AD-A069 839 BOEING AEROSPACE CO SEATTLE WA BOEING MILITARY AIRPL--ETC F/G 20/4 A BIBLIOGRAPHY OF RECENT DEVELOPMENTS IN UNSTEADY TRANSONIC FLO--ETC(U) UNCLASSIFIED FEB 79 C J BORLAND

F33615-78-C -3201

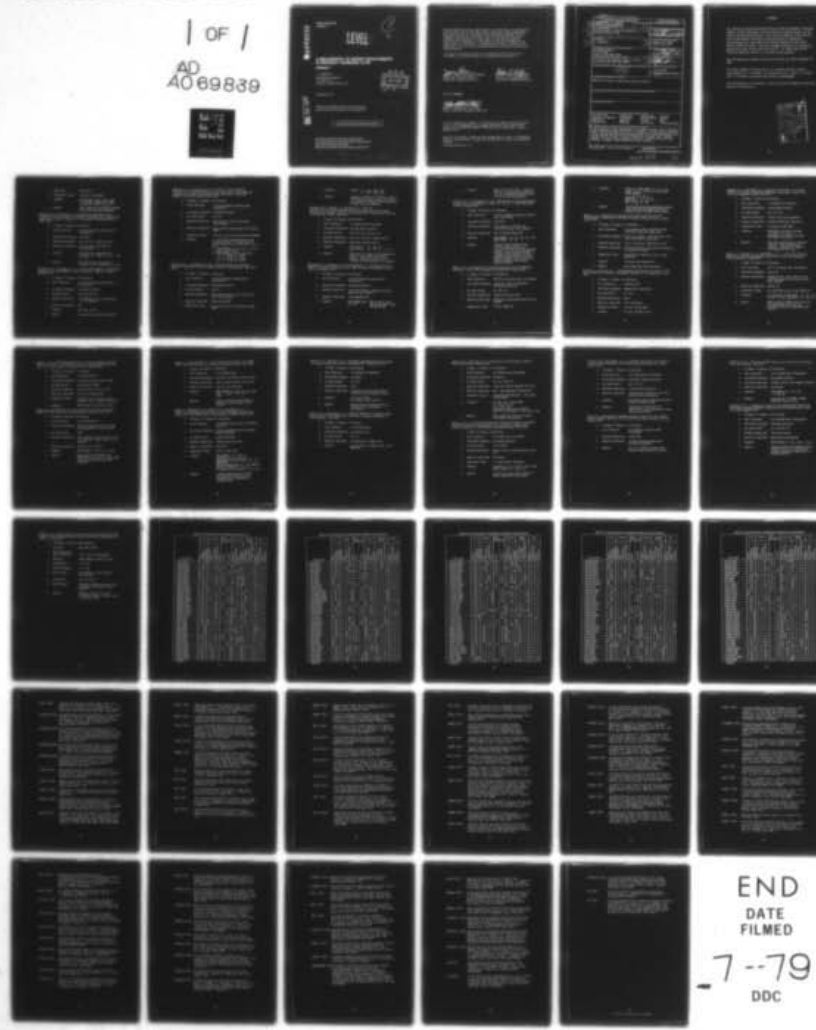

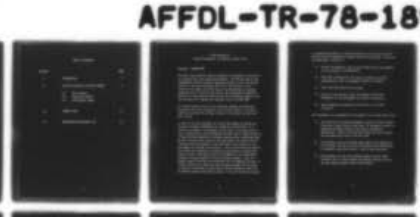

9-VOL-1
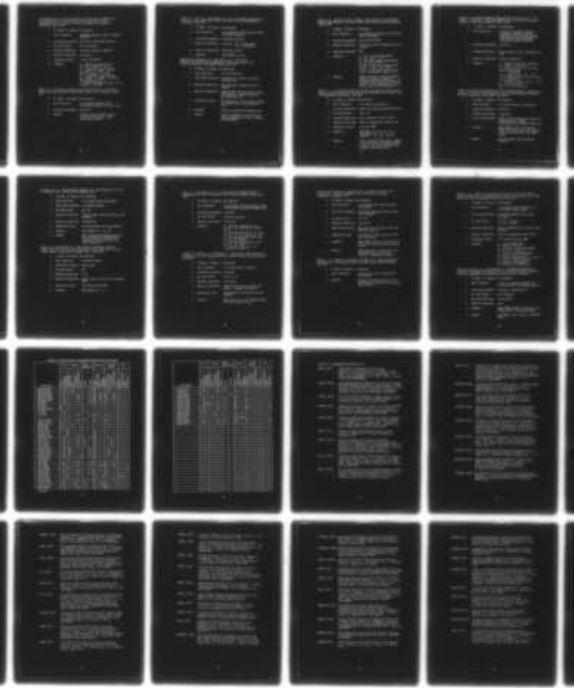
NL
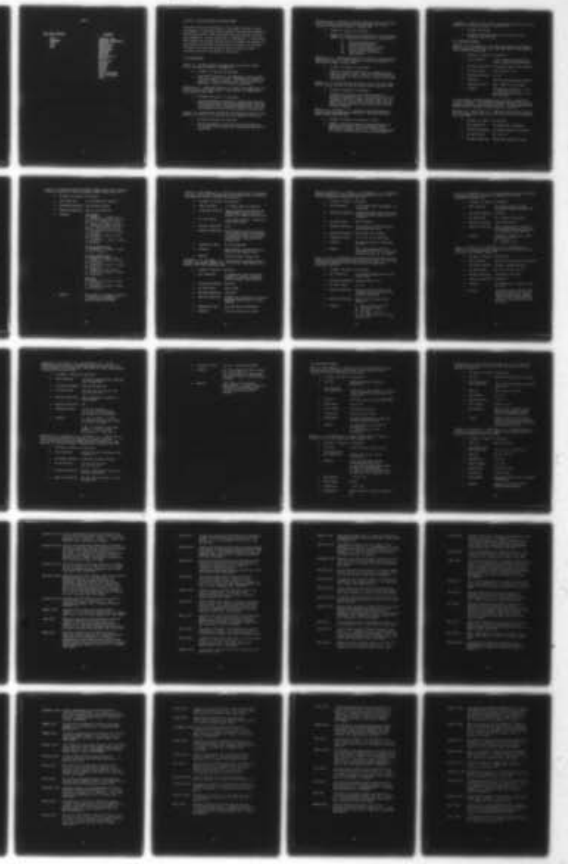

DATE

$-.79$ 


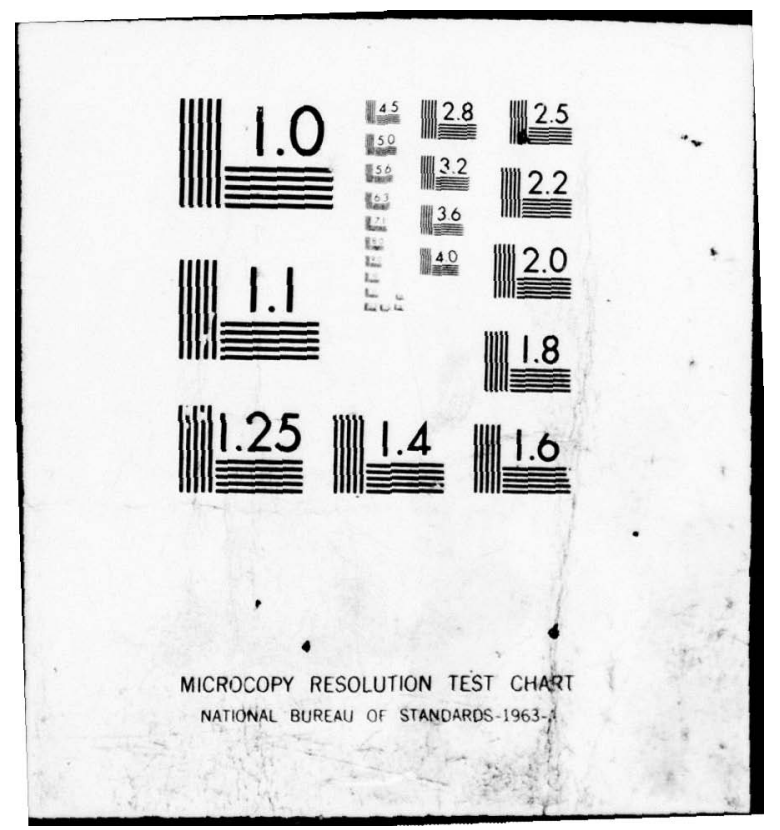


AFFDL-TR-78-189

Volume I

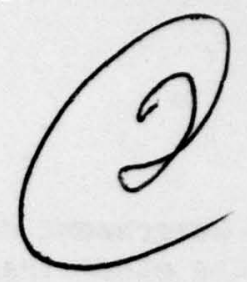

$\infty$

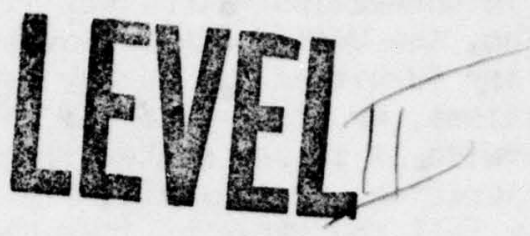

$\sqrt{c}$

\section{A BIBLIOGRAPHY OF RECENT DEVELOPMENTS IN UNSTEADY TRANSONIC FLOW}

\section{Volume I}

C. J. BORLAND

THE BOEING COMPANY

P. O. BOX 3999

SEATTLE, WASHINGTON 98124

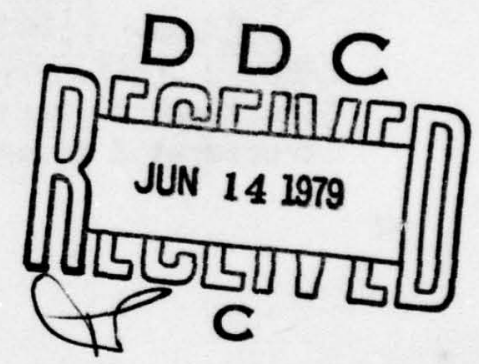

FEBRUARY 1979

TECHNICAL REPORT AFFDL-TR-78-189, Volume I

Report for Period 15 May 1978 to 15 November 1978

Approved for public release; distribution unlimited.

AIR FORCE FLIGHT DYNAMICS LABORATORY

AIR FORCE WRIGHT AERONAUTICAL LABORATORIES

AIR FORCE SYSTEMS COMMAND

WRIGHT-PATTERSON AIR FORCE BASE, OHIO 45433 
When Government drawings, specifications, or other data are used for any purpose other than in connection with a definitely related Government procurement operation, the United States Government thereby incurs no responsibility nor any furnished, or in any way supplied the said drawings, specifications, or other data, is not to be regarded by implication or otherwise as in any manner licensing the holder or any other person or corporation, or conveying any rights or permission to manufacture, use, or sell any patented invention that may in any way be related thereto.

This report has been reviewed by the Information Office (OI) and is releasable to the general public, including foreign nationals.

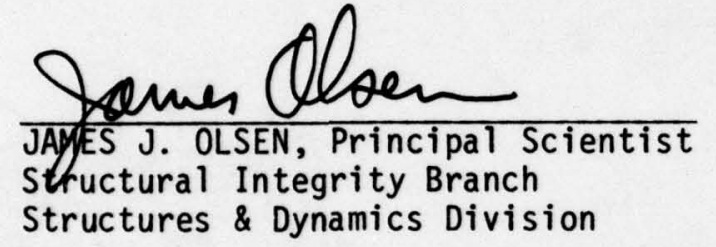

Structures \& Dynamics Division

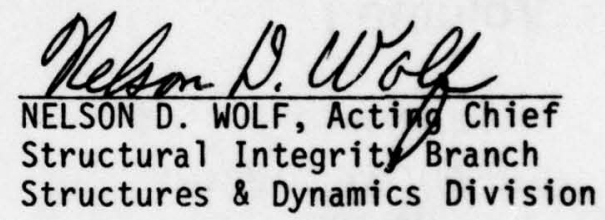

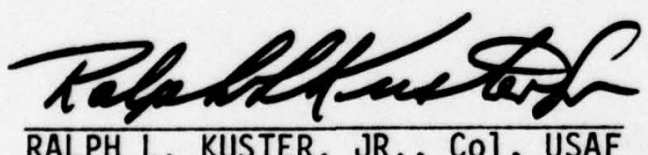

Chief, Structures \& Dynamics Division

"If your address has changed, if you wish to be removed from our mailing list, or if the addressee is no longer employed by your organization please notify AFFDL/FBE, WPAFB, $O H 45433$ to help us maintain a current mailing list."

Copies of this report should not be returned unless return is required by security considerations, contractual obligations, or notice on a specific document.

AIR FORCE/56780/9 May $1979-125$ 


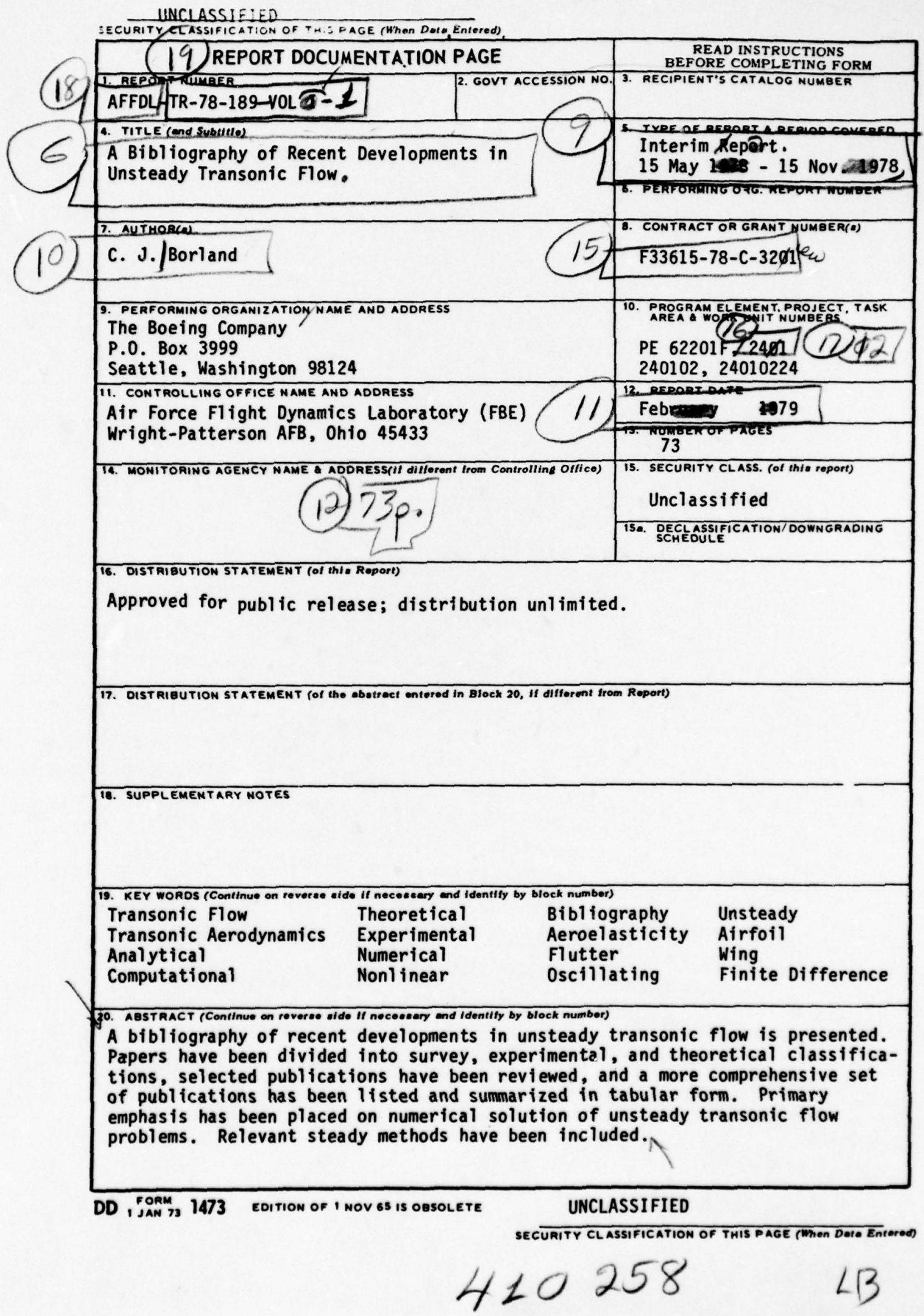


This report was prepared by Boeing Military Airplane Development, The Boeing Company, Seattle, Washington, for the Structural Integrity Branch, and the Analysis and Optimization Branch of the Structural Mechanics Division, Air Force Flight Dynamics Laboratory, Wright Aeronautical Laboratories, Wright-Patterson Air Force Base, Ohio. Boeing conducted the work under Contract F33615-78-C-3201, "Transonic Unsteady Aerodynamics for Aeroelastic Applications" under Project 2401, and Task 02 Dr. James 01 sen of the Structural Mechanics Division is the AFFDL Project Engineer.

The bibliography was prepared during the period May 15, 1978 to November 15, 1978.

The Project Manager for Boeing was Dr. H. Yoshihara and the Principal Investigator was C. J. Borland. The assistance of W. C. Chin, F. E. Ehlers, and $D$. P. Rizzetta in preparing the reviews is acknowledged.

This bibliography will be updated at yearly intervals throughout the four-year duration of the contract.

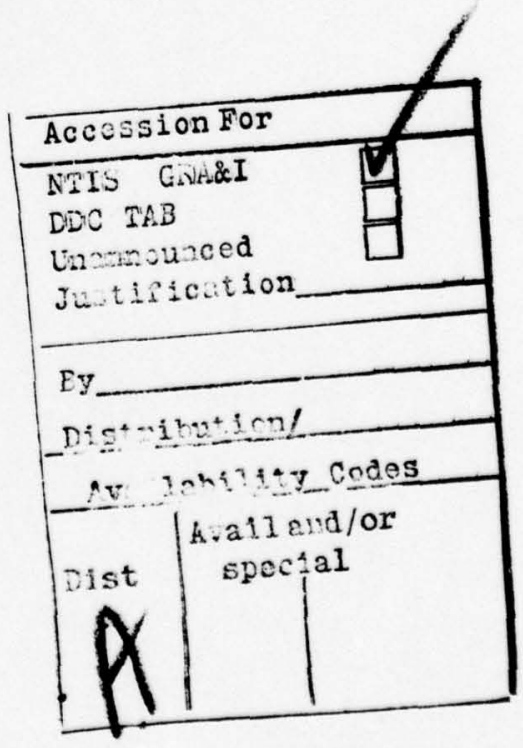


I

II

III

IV
INTRODUCTION

DETAILED REVIEWS OF SELECTED PAPERS

2.1 Survey Papers

2.2 Theoretical Papers

2.3 Experimental Papers

SUMMARY CHART

COMPREHENSIVE REFERENCE LIST
1

5

37

45 
A Bibliography of

Recent Developments in Unsteady Transonic Flow

\section{SECTION I INTRODUCTION}

The years since 1970 have seen an explosion of information in the field of transonic flow, both steady and unsteady. This has been due to two complementary and not entirely independent factors: first, the renewed interest in aircraft operations, both civil and military, in the transonic flow regime, primarily due to the availability of advanced technology airfoil sections; and second, the development of numerical methods, for the transonic nonlinear partial differential equations and the availability of appropriate computers such as the CDC 7600 .

For steady transonic flow, finite difference methods are being used extensively for design purposes in the aircraft industry. The use of numerical solutions for unsteady transonic flow however has been much more limited.

In order to lay the groundwork for further development of methods for transonic unsteady aerodynamics for aeroelastic applications, it is desirable to prepare a bibliography in the field. The primary ground rule in preparation of the bibliography has been that it should primarily include available methods for unsteady transonic flow, classifying them by their characteristic features, and those papers for steady transonic flow which have contributed, or may contribute in the future, to the development of unsteady flow solutions. Since finite difference methods have proven most promising, an attempt has been made to include those steady transonic flow papers which are fundamental to the development, extension, and application of finite difference methods. Thus, for example, papers which describe techniques such as hodograph solutions or integral methods for steady flow have not been included in the review. In addition, experimental studies which provide information useful for correlation purposes have been included. In view of the massive amount 
of information available, it has been necessary to establish additional ground rules for elimination of papers from the bibliography. These have excluded papers relating to:

a) Internal aerodynamics, such as flow through inlets and channels, or over cascades in turbomachines;

b) Flow about configurations not directly related to aircraft configurations, such as axisymmetric bodies or spheres;

c) Flows involving rotary wing aircraft;

d) Airfoil or wing design, unless the methods involved are fundamental to the development of transonic flow theory;

e) Those documents not generally accessible or in the open literature.

The development of a comprehensive bibliography list followed these lines:

1) The facilities of the Boeing Company's technical library system were used to search several data bases for documents relevant to steady and unsteady transonic flow, and to aeroelastic applications. Table 1 lists the data bases that were accessed, and the keyword descriptors that were used.

2) A preliminary sorting eliminated those papers which appeared to be irrelevant, did not fit within the ground rules stated above, or appeared as duplicate entries in two or more data bases.

3) The remaining list was cross-checked against several large reference lists in survey papers and against personal files to see that important papers had not been missed. 
4) A selected set of papers was chosen for more detailed review. An effort was made to include all those papers which would have a direct bearing on the further development of solutions for unsteady transonic flow. These detailed reviews included classification into categories of survey, theoretical, and experimental papers. For the theoretical papers, further classifications as to the basic equation used, coordinate systems employed, number of mesh points in the finite difference solutions, boundary conditions, numerical algorithms, computation times, and examples given were made. For experimental papers, the facility employed, test section and wall conditions, Mach numbers, motion types and frequencies, and measurements performed were described. If classifications have been omitted, they have not been discernible from the published paper.

5) For those papers not chosen for detailed review, a basic classification into categories of survey paper, theoretical, or experimental was performed where feasible. For theoretical papers, the basic equations, applicable geometries, and numerical methods were determined.

6) A summary chart was prepared for all of the references in the comprehensive list describing the above classifications where possible.

The following sections of this document consist of the detailed reviews for selected papers, a summary chart, and a comprehensive list of references, including author, title, source, date, and accession number (where available). 
TABLE 1

DATE BASES SEARCHED

NTIS

COMPENDEX

INSPEC

DDC

NASA

\section{KEYWORDS}

TRANSONIC FLOW

TRANSONIC AERODYNAMICS

ANAL YTICAL

COMPUTATIONAL

THEORETICAL

EXPERIMENTAL

NUMER ICAL

NONL INEAR

AEROELASTICITY

FLUTTER

OSCILLATING

UNSTEADY

PRESSURE

AIRFOIL

CONTROL

THREE DIMENSIONAL

FINITE DIFFERENCE

WING 
Using the criteria described above, various papers relating to recent developments in steady and unsteady transonic flow have been selected for more detailed review. Papers and reports have been divided into three basic categories: survey, theoretical, or experimental. In cases where there seemed to be overlap into more than one category, the category has been chosen which describes the dominant or key features of the paper. The majority of the papers chosen for detailed review are those describing theoretical development or application.

\subsection{Survey Papers}

Ashley, H.: Unsteady Subsonic and Supersonic Inviscid Flow; AGARD CP-227, Unsteady Aerodynamics, September 1977.

- 32 pages, 21 figures, 106 references

- Discussion of analysis of time-dependent inviscid external flows over shapes of interest. Formulation of mathematical problem for velocity potential where arbitrary motions are emphasized. Methods of solution are summarized.

Ballhaus, W. F.: Some Recent Progress in Transonic Flow Computation; Von Karman Institute for Fluid Dynamics, Lecture Series 87, Computational Fluid Dynamics, March 1976.

- 116 pages, 56 figures, 71 references

- Reviews development of methods for steady three-dimensional flows about wing and wing-fuselage configurations, unsteady two dimensional flows, separated turbulent flows, the finite volume method and airfoil design by numerical optimization.

Jameson, A.: Transonic Flow Calculations; Von Karman Institute for Fluid Dynamics, Lecture Series 87, Computational Fluid Dynamics, March 1976.

- 84 pages, 28 figures, 80 references

- Reviews development of numerical solution techniques for full potential and transonic small disturbance equations for steady flow. 
McCroskey, W. J.: Prediction of Unsteady Separated Flow on Oscillating Airfoils; AGARD-Lecture Series 94 Three Dimensional and Unsteady

Separation at High Reynolds Numbers, February 1978.

- 8 pages, 11 figures, 34 references

- Summary of chniques for predicting flows at high Reynolds numbers with large amounts of separation. These include:
1) Discrete potential vortex
2) Boundary layer methods
3) The strong interaction approach
4) Navier - Stokes equations
5) Correlations of existing data
6) Other methods

McCroskey, W. J.: Some Unsteady Separation Problems for Slender Bodies; AGARD-Lecture Series 94, Three Dimensional and Unsteady Separation at High Reynolds Numbers, February 1978.

- 11 pages, 16 figures, 24 references

- Summary of prediction techniques for unsteady flow over wings and rotating blades. Features of unsteady separated flows that are not simple extensions of quasi-steady flows are discussed.

Mykytow, W. J.: A Brief Overview of Transonic Flutter Problems; AGARD CP-226, Unsteady Airloads in Separated and Transonic Flow, April 1977.

- 11 pages, 12 figures, 14 references

- Introduction to Transonic Unsteady Aerodynamics for Aeroelastic Phenomena session. Reviews historical flutter incidents, trends for straight and swept wings, data for conventional vs. supercritical airfoils, wind tunnel wall effects, trends with stores and external tanks, and some nonlinear phenomena.

Mykytow, W. J., and 01sen, J. J.: A Resume of Agard SMP Meeting on Transonic Unsteady Aerodynamics; AGARD-CP-227, Unsteady Aerodynamics Ottawa, Canada, September 1977.

- 20 pages, 21 figures, 9 references, 2 tables

- Summary of specialists meeting on "Unsteady Airloads in Separated and Transonic Flow" at meeting of SMP of Agard-Lisbon, Portugal, April 1977. Outlines mutual needs and interests between aeroelasticians and aerodynamics. 
Tijdeman, H.: Remarks of the Transonic Flow Around Oscillating Airfoils; AGARD-CP-227 Unsteady Aerodynamics, September 1977.

- 52 pages, 30 figures

o keview of recent experimental and numerical results (graphical material only)

\subsection{Theoretical Papers}

Baldwin, B. S., and Lomax, H.: Thin Layer Approximation and Algebraic Model for Separated Turbulent Flows; AIAA Paper 78-257, 16th Aerospace Science Meeting, January 1978.

- 8 pages, 11 figures, 13 references

- Basic Equations: $2 \mathrm{D}$ thin layer equations NavierStokes, algebraic turbulence model

- Coordinate System(s): Transformed, Body fitted, Stretched

o No. Mesh Points: $\quad \star 64 \times 36$ and $77 \times 36$

- Boundary Conditions: No slip

- Algorithm: Implicit approximate factorization

- Computation Times: $1400-6900 \mathrm{sec}$, on CDC 7600

- Examples:

$$
\begin{aligned}
& \text { Garbedian-Korn Airfoil, } M=.756, \\
& \quad=2.66, \operatorname{Re}=21 \times 106 \\
& 18 \% \text { Circular Arc, M }=.783, \alpha=0, \\
& \operatorname{Re}=11 \times 106
\end{aligned}
$$

* For 2-D papers, the mesh dimensions are given for streamwise and vertical directions. For 3-D papers, the mesh dimensions are given for streamwise, spanwise, and vertical directions. If total number of mesh points is given, individual directions were not available.

Ballhaus, W. F., and Bailey, F. R.: Numerical Calculation of Transonic Flow About Swept Wings; AIAA Paper 72-677; 5th Fluid and Plasma Dynamics Conf., July 1972.

- 10 pages, 11 figures, 13 references

- Basic Equations: 3-D Steady small disturbance

- Coordinate System(s): Stretched, Sheared, 3-D Cartesian

- No. Mesh Points: $68 \times 30 \times 49$

- Boundary Conditions: Steady wing, Klunker Far-Field 

- Algorithm:
Relaxation
- Computation Times:
4-6 hours, IBM $360 / 67$
o Examples:
C141 Airfoil section $M=.752$,
$\alpha=00 ; M=.853, \alpha=00,20$
23.750 Swept const. chord wing;
o Remarks:
Basic paper for 3-D steady transonic flow by Bailey-Ballhaus method.

Ballhaus, W. F., and Lomax, H.: The Numerical Simulation of Low Frequency Unsteady Transonic Flow Fields; Proceedings 4 th International Conference on Numerical Methods in Fluid Dynamics (Springer-Verlag, $1974), 1973$.

- 7 pages, 6 figures, 2 references

- Basic Equations: 2-D Low frequency unsteady small disturbance

- Coordinate System(s): Cartesian

- Boundary Conditions: Solid airfoil, Impulsive start

- Numerical Algorithm: SLOR (steady); Semi-implicit

- Examples: Parabolic Arc (time-dependent thickness) $M=.785, \delta=.1 ; M=.80$, $\mathrm{k}=.03$

- Remarks: Preliminary work on unsteady transonic finite difference solutions

Ballhaus, W. F., and Steger, J. L.: Implicit Approximate - Factorization Schemes for the Low-Frequency Transonic Equation: NASA TM X-73,082, November 1975.

- 41 pages, 17 figures, 12 references

- Basic Equations: 2-D Low frequency unsteady small disturbance

- Coordinate System(s): Stretched Cartesian

o No. Mesh Points: Typically $80 \times 80$

- Boundary Conditions: Low frequency, $\phi_{X}=0$ downstream,

- Numerical Algorithm: $A D I$

- Examples: Pulsating airfoil

- Remarks: Preliminary feasibility study only 
Ballhaus, W. F., and Goorjian, P. M.: Implicit Finite Difference Computations of Unsteady Transonic Flows About Airfoils, Including the Treatment of Irregular Shock Wave Motions, AIAA Paper 77-205, Los Angeles, California, January 24-26, 1977.

- 10 pages, 9 figures, 17 references

- Basic Equations: 2-D, low frequency unsteady small disturbance

- Coordinate System(s): Stretched Cartesian

o No. Mesh Points: $99 \times 79$

- Boundary Conditions: Time accurate, small disturbance, low-frequency

- Numerical Algorithm: Fully implicit approximate factorization

- Computation times: $8 \mathrm{sec}$ per cycle of oscillation on CDC7600

- Examples:

1. Oscillatory plunging airfoil (no designation given) - comparison with linear theory; $M=0.7,0.8,0.9 ; 0<k<$ 0.4

2. NACÁ 64A006, $k=0.1,0.8 M<0.9$ - pitching oscillation

3. NACA 64A006, trailing edge flap

i) $k=0.064, M=0.80$

ii) $k=0.234, M=0.875$

iii) $k=0.179, M=0.852$

Ballhaus, W. F., and Goorjian, P. M.: Efficient Solution of Unsteady Transonic Flows About Airfoils, AGARD Conference Proceedings No. ?26, April 1977.

- 11 pages, 9 figures, 7 references

- Basic Equations 2-D low frequency unsteady small disturbance

- Coordinate System(s): Stretched Cartesian

o No. Mesh Points: $\quad 99 \times 79$

- Boundary Conditions: Oscillating airfoil, oscillating .25c flap; free response

- Numerical Algorithm: Time-marching ADI

- Computation Times: $8 \mathrm{sec}$ per cycle of oscillation on (CDC $7600)$ 


\begin{tabular}{ll}
$0 \quad$ Examples: & $65 A 006 \quad \begin{array}{l}M=.875, .854, .822 \\
k=.234, .179, .248\end{array}$ \\
& \multicolumn{1}{l}{$\begin{array}{l}\text { Compares results with Tijdeman's data for } \\
\text { oscillating control surface, and solves } \\
\text { one degree of freedom flutter problem for } \\
\text { various levels of damping. }\end{array}$}
\end{tabular}

Ballhaus, W. F., Jameson, A., and Albert, J.: Implicit Approximate-Factorization Schemes for the Efficient Solution of Steady Transonic Flow Problems, Paper at AIAA Computational Fluid Dynamics Meeting, (a)so NASA TMX 73-202), June 1977.

- 8 pages, 10 figures, 20 references

- Basic Equations: 2-D steady small disturbance

- Coordinate Systems(s) Variable mesh cartesian

o No. Mesh Points: $128 \times 32 ; 43 \times 32 ; 22 \times 16$

o Boundary Conditions: Free jet, free air; steady airfoil

- Numerical Algorithm: Approximate factorization

- Examples: $\quad 10 \%$ parabolic $M=.84, .90$

Korn airfoil $M=.75 \quad \alpha=.5,1.0$

- Remarks: Shows that AF scheme is approximately 10

times as fast as SLOR for similar results. (NASA TMX 73-202 version includes only $10 \%$ parabolic arc example)

Ballhaus, W. F., Goorjian, P. M., and Yoshihara, H.: Unsteady Force and Moment Alleviation in Transonic Flow, AGARD Conference Proceedings No. 227, Paper 14, September 1977.

010 pages, 15 figures, 3 references

- Basic Equations: 2-D low frequency unsteady small disturbance

o Coordinate System(s): Stretched Cartesian

- Boundary Conditions: Pitch oscillations, leading \& trailing edge flap oscillations

- Numerical Algorithm: Time dependent ADI

- Examples:

NACA 64 A006 with $M=.85$
10\% LE and TE flaps

25\% LE and $15 \%$ TE flaps $10 \%$ chord bump 
- Remarks:

Application of Ballhaus - Goorjian methor to demonstrate possibilities of lift and moment cancellation by control surface deflections.

Ballhaus, W. F., and Goorjian, P. M.: Computation of Unsteady Transonic Flows by the Indical Method, J. AIAA, Vol. 10, No. 2, (also AIAA Paper 77-447), February 1978.

- 8 pages, 10 figures, 12 references

- Basic Equations:

2-D low frequency unsteady transonic small disturbance

- Coordinate System(s): Cartesian

- Boundary Conditions: Solid airfoil, indicial and sinusoidal motion, free response

- Numerical Algorithm: ADI following steady SLOR

- Examples:

NACA $64 A 006 \quad M=.8, .85, .87, .88$ NACA $64 A 010$

$M=.8$

- Remarks:

Compares time integration and indicial method results for sinusoidal motions and step function motions. Also solves one degree of freedom flutter equation by time integration with various values of damping, demonstrating subcritical, neutraliy stable, and unstable dynamic behavior.

Boppe, C. W.: Calculation of Transonic Wing Flows by Grid Embedding, AIAA Paper 77-207; 15th Aerospace Sciences Meeting, January 1977.

- 11 pages, 17 figures, 17 references

- Basic Equations: Modified 3-D steady small disturbance

- Coordinate System(s): Cartesian course, transformed Cartesian fine grids

- No. Mesh Points: $51 \times 26 \times 31$

o Boundary Conditions: Steady wing, free field

- Numerical Algorithm: Relaxation (alternating course \& fine sweeps)

- Computation Times: $10 \mathrm{~min}$, CYBER 175 
0

Examples:

- Remarks:
Airfoils: NACA $63 A 006 \quad M=.9 \alpha=10$

Wings: $A R=4$, const chord 350 swept (NACA 63A006)

$M=.9 \quad \alpha=3$

ONERA M6, $M=.84 \quad \alpha=3$

RAE wing $A \quad M=.9 \quad \alpha=1$

TACT 1 wing $M=.85$

Extends Bailey-Ballhaus method by including a fine grid and adding additional spanwise terms for swept shock capture.

Boppe, C. W.: Computational Transonic Flow About Realistic Aircraft Configurations, AIAA Paper 78-104 16th Aerospace Sciences Meeting, January $16-18,1978$, .

010 pages, 19 figures, 14 references

- Basic Equations: 3 D transonic small disturbance with additional terms for swept wings

- Coordinate System(s): Three grid system: Fine body grid, wing grid, and global coarse grid.

- Boundary Conditions: Exact on body and linearized on wing

- Numerical Algorithm: Relaxation method with upwind diffarencing for hyperbolic points

- Computation Times: 45 minutes on IBM 370,15 min. on CDC CYBER 175

- Examples: Two wing body combinations

- Remarks: Good comparison with experiment.

Burstein, S. and Mirin, A.: Time Dependent Calculations for Transonic Flow, 3rd International Conference on Numerical Methods in Fluid Mechanics, July 1972.

- 12 pages, 2 figures, 10 references

- Basic Equations Unsteady Euler

- Coordinate System(s): Mapped polar coordinates

- No. Mesh Points: $15 \times 80$

- Boundary Conditions: Exact

- Numerical Algorithm: Time integration

- Computation Times: $1 / 2$ hour on CDC 7600

- Examples: Ellipse, and NAE airfoil 
Caughey, D. A., and Jameson, A.: Numerical Calculation of Transonic Potential Flow About Wing-Body Combinations, AIAA Paper 77-677, 10th Fluid \& Plasmadynamics Conf, June 1977.

- 8 pages, 7 figures, 29 references

- Basic Equations 3 D steady full potential

- Coordinate System(s): Transformed Cartesian

o No. Mesh Points: $96 \times 16 \times 16$

- Boundary Conditions: Steady wing (exact geometry)

- Numerical Algorithm: Rotated difference relaxation

- Computation Times: 7 minutes on $\operatorname{CDC} 7600$

- Examples: ONERA M6 on circular fuselage

$M=.839, \alpha=3.070$

ONERA M6 on area-ruled fuselage

$M=.839, \alpha=3.070$

- Remarks: Describes organization of computer code for large 3-dimensional problems. Extension of Jameson full-potential 3-D code.

Chen, A. W., Dickson, L. J., and Rubbert, P. E.: A Far Field Matching Method for Transonic Computations, AIAA Paper 77-203, 15th Aerospace Sciences Meeting, January 1977.

- 7 pages, 9 figures, 10 references

- Basic Equations 2-D \& 3-D steady small disturbance

- Coordinate System(s): Cartesian

o Boundary Conditions: Steady airfoil, wing; new far field condition based on 1st and 2nd order panel methods

- Numerical Algorithm: Relaxation

- Computation Times: $3-D ; 800-1300$ CP sec on CDC CYBER 175

- Examples: 2-D asymmetric $6 \%$ parabolic $M=.77 \quad \alpha=0$

3-D AR $=6$ rect, NACA $0012 \quad M=.82 \quad \alpha=0$

- Remarks: Modification of Murman-Cole (2-D) and Bailey-Ballhaus (3-D) for new far-field condition; comparison with Klunker. 
Cunningham, A. M. Jr.: An Oscillatory Kernnel Function Method for Lifting Surfaces in Mixed Transonic Flow, AIAA Paper 74-359, AIAA/ASME/SAE 15th Structure, Structural Dynamics and Materials Conference, April 1974.

- 11 pages, 11 figures 12 references

- Basic Equations downwash-pressure function integral equation

- Coordinate System(s): Not finite difference solution

- No. Mesh Points: 24 or 33 panels

- Boundary Conditions: Unsteady, general frequency

- Numerical Algorithm: No details

- Computation Times: 1 min. IBM 370/155

- Examples: 1. TND-344 rectangular wing

$A R=3, M=0.90, k=0.13$

2. Trapezoidal wing of Becker

$M=0.937, k=0.218, \quad \alpha=0$

Also $M=0.997, k=0.207$

3. Trapezoidal wing of Becker, $M=0.942, k=0.386, \alpha=0$ with oscillating aileron

4. TND-344 rectangular wing, $R=3$, steady $M=0.9,1.0$

Dowell, E.: A Simplified Theory of Oscillating Airfoils in Transonic Flow: Review and Extension, AIAA Dynamic Specialists Conference, March 1977.

- 42 pages, 12 figures, 30 references

- Basic Equations 2 D unsteady transonic small disturbance equation with $\phi_{t t}$ term.

- Coordinate System(s): Cartesian

- Remarks:

Analytic study related to local linearization concept. Good qualitative results. 
Fung, K. Y., Yu, N. J., and Seebass, R.: Small Unsteady Perturbations in Transonic Flows, AIAA Paper 77-675, AIAA 10th Fluid \& Plasmadynamics Conference, June 1977.

- 12 pages, 10 figures, 18 references

- Basic Equations Low frequency, time linearized small disturbance equation

- Coordinate System(s): Stretched Cartesian

- Boundary Conditions: low freq; $\phi_{x}=0$ downstream

$\phi=0$ elsewhere

- Numerical Algorithm: ADI/shock fitting

- Examples: NACA $64 A 006$ in pitch

Hafez, M. M., Murman, E. M., and South, J. C.: Artificial

Compressibility Methods for Numerical Solution of Transonic Full

Potential Equation, AIAA Paper 78-1148, July 1978.

- 9 pages, 11 figures, 16 references

- Basic Equations

2 D full potential

- Coordinate System(s): Cartesian with velocity oriented differencing

- Boundary Conditions: Not discussed, probably similar to Jameson

- Numerical Algorithm: Jamesons SOR, ADI and Explicit using Star computer. Damping added by modifying density.

- Computation Times: ADI 300 cycles in 38 seconds on CYBER 175,3000 cycles in 14 seconds on Star.

- Examples:

Circular cylinder and parabolic arc.

- Remarks:

Tests 3 different finite difference solution methods on a vector computer (CDC STAR 100). 
Henne, P. A., and Hicks, R. M., Transonic Wing Analysis Using Advanced Computational Methods, AIAA Paper 78-105, 16th Aerospace Science Meeting, January 1978.

- 9 pages, 19 figures, 9 references

- Basic Equations

3-D steady full potential; 3-D steady small disturbance

- Coordinate System(s): Stretched cartesian

- Boundary Conditions: Solid wing; small disturbance \& exact surface

- Numerical Algorithm: SOR

- Examples:

1. $A R=10$, RECT (NACA 0010)

$M=.75, .85, \quad \alpha=0$

2. $A R=6.330$ swept (NACA 64-212)

$M=.6, .85 \alpha=40$

3. $A R=7.320$ swept supercritical

(14\%) $M=.84, \quad \alpha=1.850$

4. $A R=7.320$ swept supercritical

(14\%) - modified $M=.84 \quad \alpha=20$

o Remarks:

Compares results of Bailey-Ballhaus, Boppe, and Jameson-Caughey for transport-type wings, concludes full potential gives substantially better answers than small-disturbances.

Holst, T. L.: An Implicit Algorithm for the Conservative Transonic Full Potential Equation Using An Arbitrary Mesh, AIAA Paper 78-113, 11th Fluid \& Plasmadynamics Conference, July 1978.

- 12 pages, 8 figures, 19 references

- Basic Equations 2-D steady full potential

- Coordinate System(s): Numerically generated conformal mesh

o No. Mesh Points: $149 \times 28$

- Boundary Conditions: Exact steady on airfoil surface

- Numerical Algorithm: Implicit approximate factorization

- Computation Times: $6 \mathrm{sec}$ CDC 7600

- Examples:

NACA $0012 M=.63, .75 \quad \alpha=2$

NACA $64 A 410 \quad M=.72 \quad \alpha=0$

$75-06-12 \quad M=.75 \quad \alpha=.12$

- Remarks:

Shows substantial decrease in computation time over line overrelaxation schemes. Describes techniques for numerical mesh generation. 
Isogai, K.: Unsteady Transonic Flow Over Oscillating Circular - Arc Airfoils, AIAA Paper 74-360 AIAA/ASME/SAE 15th Structures, Structural Dynamics, \& Materials Conference, April 1974.

- 11 pages, 11 figures, 16 references

- Basic Equations

3-D small disturbance, general frequency, potential integral equation; kernel function assumed with harmonic time dependence linearized unsteady criterion

- Coordinate System(s): Cartesian

- Boundary Conditions: High frequency, small disturbance on body

- Numerical Algorithm: Gaussian Quadrature

- Examples:

1. Steady circular arc $-6 \%$ thick $M=1.011$, $\alpha=00(2-0)$ 2. Circular arc, $M=1.0$, pitch about leading edge $0<\mathrm{k}<1.0$ $t / c=0.025,0.05,0.10 \quad 2-0$ 3. circular arc, $M=1.0, k=0.10$ $t / c=0.025,0.10$

4. circular arc, $A R \quad 2.52, M=1.2$, $k=1.0, t / c=0.025$ and $k=2.3, t / c=0.10$

Isogai, K.: Approximate Method for Calculating Aerodynamic Loadings on an Airfoil Oscillating in High Subsonic Flow, Technical Report of National Aerospace Laboratory, NAL TR-455T, May 1976.

o 11 pages, 7 figures, 14 references

- Basic Equations

2 D linearized small distrubance

- Coordinate System(s): Cartesian

- Boundary Conditions: Linearized

- Numerical Algorithm: Kernel function method with numerical integration and a simple approximation of the mean steady flow

- Examples:

NACA $64 A 006$ airfoil with quarter chord oscillating flap, and complete airfoil oscillating in pitch about center.

o Remarks:

Good agreement with experimental results. 
Isogai, K.: Numerical Study on Unsteady Transonic Flow over Oscillating Airfoils Using the Full Potential Equation, NASA TP-1120, April 1978.

- 59 pages, 21 figures, 9 references

- Basic Equations 2-D, unsteady full potential

- Coordinate System(s): non-uniform Cartesian

- Numerical Algorithm: Explicit time marching

- Examples:

NACA $64 A 006$

1. steady, $M=0.875 \quad a=0$

2. $1 / 4$ chord flap oscillation, $M=$

$0.875, k=0.234, B=10 \sin \omega t$.

3. steady, $M=0.860, \alpha=00$

4. $1 / 4$ chord flap oscillation, $M=$

$0.86, k=0.234, B=10$ sin $\omega t$

5. pitching, $M=0.71, k=0.10$,

$\alpha=10 \sin \omega t$

6. pitching, $M=0.70, k=0.10$,

$\alpha=10 \sin \omega t$

7. pitching, $M=0.68, k=0.10$,

$\alpha=10 \sin \omega t$

70-10-13 supercritical

1. pitching, $M=0.68, k=0.10$,

$\alpha=10+10 \sin \omega t$

79-03-12 supercritical

1. steady, $M=0.79, \alpha=00$

2. pitching, $M=0.75, k=0.30$,

$\alpha=10 \sin \omega t$,

3. pitching, $\dot{M}=0.75, k=0.10$,

$\alpha=10 \sin \omega t$,

4. steady, $M=0.75, \alpha=00$

5. pitching, $M=0.70, k=0.10$,

$\alpha=10 \sin \omega t$,

NACA 0012

1. pitching, $M=0.79, k=0.10$,

$\alpha=10 \sin \omega t$

2. pitching, $M=0.70, k=0.10$,

$\alpha=10 \sin \omega t$

Remarks:

This report is a summary of results, no theoretical development or numerical details provided. 
Jameson, A., and Caughey, D. A.: Numerical Calculation of the Transonic Flow Past a Swept Wing, ERDA Research and Development Report, Mathematics and Computing, report no. C00-3077-140, June 1977.

- 93 pages, 14 figures, 18 references

- Basic Equations

3 D steady state full potential

- Coordinate System(s):

Swept coordinates aligned with the leading edge. Nearly conformal mapping in streamwise cross sections.

o No. Mesh Points:

Large number possible. Program uses out of core solver.

- Boundary Conditions: Exact

- Numerical Algorithm: Row relaxation, with flow oriented differencing and upwind differencing at supersonic points. Uses coarse and fine grids. Non-conservative differencing.

- Computation Times: No times presented

- Examples:

Good agreement with measurements on ONERA wing and Douglas wing.

- Remarks:

Complete program listing given.

Klineberg, J. M., and Steger, J. L.: Calculation of Separated Flows at Subsonic and Transonic Speeds, Proc. 5th Int. Conf. Num. Methods Fluid Dynamics, June 1976, Springer-Verlag.

- 9 pages, 8 figures, 7 references

- Basic Equations 2 d dimensional small disturbance equations in velocity components. Integral boundary layer equations.

- Coordinate System(s): Cartesian

- No. Mesh Points: 2300 to 3800

- Boundary Conditions: Linearized

- Numerical Algorithm: Standard over relaxation for inviscid equations, Runge Kutta for viscous equations

- Computation Times: Less than 30 min of IBM 360-67

- Examples: Flow over circular arc airfoil 
Lee, K. D., Dickson, L. J., Chen, A. W., and Rubbert, P. E.: An Improved Matching Method for Transonic Computations, AIAA Paper 78-ii16, 11 th Fluid \& Plasmadynamics Conference, July 1978.

o 5 pages, 7 figures, 4 references

- Basic Equations $3 \mathrm{D}$ transonic small disturbance; $3 \mathrm{D}$ linearized

- Coordinate System(s): Stretched Cartesian near-field finite element mid-field singularities on far field

- No. Mesh Points: 9072 to 35,840

o Boundary Conditions: Solid airfoil; midfield matching; panel method far field

o Numerical Algorithm: Relaxation; finite element

- Computation Times: 231 to 749 CP sec (CDC 7600)

- Examples: $\quad$ Rectangular wing AR $=6$ NACA 0012 $M=.82$

0 Remarks:

Adds finite-element mid field representation to far field approach described in Chen, et. al. (1977).

Levy, J. J. Jr.: An Experimental and Computational Investigation of the Steady and Unsteady Transonic Flow Field About an Airfoil in a Solid Wall Test Channel, AIAA Paper 77-678, 10th Fluid and Plasmadynamics

Conference, June 1977.

- 11 pages, 10 figures, 11 references

- Basic Equations 2 D unsteady compressible turbulent Navier-Stokes

- Coordinate System(s): Conformal about airfoil

o No. Mesh Points: $78 \times 35$

- Boundary Conditions: No slip on airfoil surface, flow tangency on wind tunnel walls, no gradients downstream

- Numerical Algorithm: Diewert code for Navier Stokes equations

- Examples:

1. Korn- Garabedian airfoil, $M=0.750, \alpha=-1.590$, $\mathrm{Re}=21 \times 106$

2. $18 \%$ thick circular arc, $\operatorname{Re}=11 \times 106, \alpha=0 \mathrm{M}=0.783$, $0.720,0.754$ 
Liu, D. D., and Winther, B. A.: Towards a Mixed Kernel Function Approach for Unsteady Transonic Flow Analysis, AGARD-CCP-227 Unsteady

Aerodynamics, September 1977.

- 17 pages, 12 figures, 49 references

- Basic Equations

3-D unsteady linearized small disturbance harmonic decomposition

- Coordinate System(s): Cartesian

$0 \quad$ No. Mesh Points: $10 \times 9$ panels per semi-wing

- Boundary Conditions: linearized on panel

- Numerical Algorithm: Gaussan Quadrature of "transonic" (only subsonic and supersonic cases are considered) kernel function

- Examples:

Rectangular unswept wing - aspect ratios of $2,3.6,4$

$M=0,5 / 3$,

$M=0.24, k=0.47$

Lomax, H., Bailey, F. R., and Ballhaus, W. F.: On the Numerical

Simulation of Three Dimensional Transonic Flow With Application to the C-141 Wing, NASA TND-6933, August 1973.

- 50 pages, 18 figures, 19 references

- Basic Equations Modified 3-D steady small disturbance

- Coordinate System(s): Sheared, stretched Cartesian

o No. Mesh Points: $68 \times 23 \times 49: 82 \times 49 \times 49$

o Boundary Conditions: Steady wing, free field

o Numerical Algorithm: Relaxation

- Examples: $\quad C$-141 wing $(A R=8$, sweep $=25.60)$ $M=.825$

o Remarks:

Develops modified transonic small disturbance equation for improved capture of swept shocks compares results of basic Bailey-Ballhaus method with wind tunnel and flight test data. 
Magnus, R. J.: Computational Research on Inviscid, Unsteady, Transonic Flow Over Airfoils - Final Report, Office of Naval Research (Code 438) Dept. of the Navy Report no. CASD/LVP 77-010, January 1977.

- 67 pages, 21 figures, 7 references

- Basic Equations 2 D unsteady Euler

- Coordinate System(s): Coordinate system fixed on body

- No. Mesh Points: Approximately 6,000

- Boundary Conditions: Exact on mean airfoil surface

- Numerical Algorithm: Explicit time-machining

- Computation Times: As long as 6 to 7 hours on some examples. Not a production program

- Examples: NACA 64A410 oscillating in pitch

Magnus, R., and Yoshihara, H.: The Transonic Oscillating Flap, AIAA Paper 76-327; 9th Fluid and Plasma Dynamics Conference, July 1976.

- 13 pages, 9 figures, 8 references

- Basic Equations

2 D unsteady Euler

o Coordinate System(s): system embedded in Cartesian mesh

No. Mesh Points: 5484

o Boundary Conditions:

Exact applied at mean location, .25 C flap includes viscous ramp at shock location

o Numerical Algorithm:

Explicit Time marching

- Examples:

NACA $64 A 006 \mathrm{M}=.875 \mathrm{k}=0, .234$

o Remarks:

Comparison with Tijdemann data demonstrates effects of viscous wedge approximation and aft specified pressures. 
Maanus, R., and Yoshihara, H.: The Transonic Oscillating Flap, AGARD CP-226, Unsteady Airloads in Transonic and Separated Flow, April 1977.

$0 \quad 5$ pages, 8 figures, 2 references

- Basic Equations 2 D unsteady Euler

- Coordinate System(s): Multiple, including surface fitted

- Boundary Conditions: Exact on mean surface, free field

- Numerical Algorithm: Explicit time marching

0 Examples: $\quad$ NACA $64 A 006$ w/ $.25 \mathrm{c}$ flap, $M=.854$, $.875,, .900 \alpha=0, \alpha=+1, k=$ .234 ,

- Remarks: $\quad$ Adds aft pressure prescription to results of previous paper (AIAA 76-327).

Mason, W., Mackenzie, D. A., Stern, M. A., and Johnson, J. K.: A Numerical Three-Dimensional Viscous Transonic Wing-Body Analysis and Design Tool, AIAA Paper 78-101; 16th Aerospace Sciences, January 1978.

- 16 pages, 11 figures, 42 references

- Basic Equations

3 D steady modified small disturbance equation

o Coordinate System(s): Multiple embedded grids (stretched, swept (artesian)

o No. Mesh Points: $\quad 35,400$ (maximum)

- Boundary Conditions: Steady wing, free field

o Numerical Algorithm: Relaxation

- Computation Times: 10 min on CDC 7600

- Examples:

NACA $64 A 010 M=.84 \alpha=0$

M6 ONERA wing $M=.92 \alpha=0$

NACA TND712 wing-body model $M=.94$

(RMA55B21)

NACA wing-body model $M=.94$ (TND712)

Advanced fighter $M=.9 \alpha=7$

$F-8$ Supercritical wing $M=.9, \alpha=3.5$

o Remarks:

Extends Bcppe method to include fuselage and viscous-inviscid interactions by including 3-D boundary layer. 
Melnik, R. E., and Ives, D. G.: On Viscous and Wind-Tunnel Wall Effects in Transonic Flows Over Airfoils, AIAA Paper No. 73-660, July 1973.

- 16 pages, 8 figures, 12 references

- Basic Equations 2-D steady full potential

- Coordinate System(s): Cartesian

o No. Mesh Points: $32 \times 92$

- Boundary Conditions: Exact

- Numerical Algorithm: Standard relaxation with upwind differencing at supersonic points.

- Examples: $\quad 17$ cases on same Korn supercritical airfoil

- Remarks: Relaxes Kutta conditions and uses measured lift to determine circulation.

Mirin, A. A., and Burstein, S.: Difference Methods for Transonic Flows About Airfoils, 4th International Conference on Numerical Methods in Fluid Dynamics, June 1974.

- 10 pages, 4 figures, 2 references

- Basic Equations 2-D unsteady Euler

- Coordinate System(s): Transformed polar

o No. Mesh Points: $80 \times 21$

- Numerical Algorithm: Time maching to steady limit

- Examples: A Korn airfoil, an NAE airfoil, and a NACA 0012 
Murman, E. M., and Cole, J. D.: Calculation of Plane Steady Transonic Flows, AIAA Journal, January 1971.

- 8 pages, 12 figures, 14 references

- Basic Equations 2-D steady small disturbance

- Coordinate System(s): Cartesian

o No. Mesh Points: $\quad 74 \times 41,148 \times 71$

- Boundary Conditions: Linear on airfoil, doublet far-field

- Numerical Algorithm: SOR - type dependent differencing

- Computation Times: Typically 400 iterations - 30 minutes on IBM $360 / 44$

- Examples: Circular Arc, curved plate, $\mathrm{K}=2.5,2.3,2.1$, $1.8,1.45,1.15$, (transonic similarity parameter) NLR $0.12-0.70-0.00 \mathrm{~K}=1.71,1.82,1.60$

o Remarks:

Basic paper for relaxation method with mixed differencing.

Murman, E. M.: A Relaxation Method for Calculating Transonic Flows With Detached Bow Shocks, Proceedings of 3rd International Conference on Numerical Methods in Fluid Mechanics (Springer-Verlag, 1973), 1972.

04 pages, 6 figures, 9 references

- Basic Equations 2-D steady small disturbance

- Coordinate System(s): Stretched Cartesian

- No. Mesh Points: 2100 to 6700

- Boundary Conditions: Steady airfoil; perforated wall, free air

o Numerical Algorithm: Relaxation

- Computation Times: .8 to 69 minutes IBM $360 / 67$

- Examples: $\quad$ Parabolic Arc, $M=1.011,1.052,1.083$ NACA 4-digit, $M=1.10,1.25$

o Remarks:

Extension of basic Murman method to handle supersonic free stream. 
Murphy, W. D., and Malmuth, N. D.: A Relaxation Solution For Transonic Flow Over Three-Dimensional Jet-Flapped Wings, AIAA Paper No. 76-98, January 1976.

- 10 pages, 7 figures, 13 references

- Basic Equations 2-D steady small distrubance

- Coordinate System(s): Stretched, sheared Cartesian

o No. Mesh Points: $68 \times 35 \times 36$

o Boundary Conditions: Linearized on wing plane

- Numerical Algorithm: Standard over-relaxation with fully conservative differencing

- Examples:

Rectangular wing, swept wing, tapered wing with ONERA section

- Remarks: Comparison of calculated lift coefficients with experimental values show good agreement.

Nixon, D.: Calculation of Unsteady Transonic Flows Using The Integral Equation Method, AIAA Paper 78-13, AIAA 16th Aerospace Sciences Meeting, January 1978.

- 11 pages, 4 figures, 13 references

- Basic Equations

2-D unsteady linearized small disturbance

- Boundary Conditions: Linearized

- Numerical Algorithm: Integral equation method with strained coordinates

o Remarks:

This is an analytic method which requires later numerical solution. 
Rizzetta, D. P.: Transonic Flutter Analysis of Two-Dimensional Airfoil, AFFDL TM-77-64-FBR, July 1977.

- 29 pages, 4 figures, 10 references

- Basic Equations

2-D unsteady small disturbance

- Coordinate System(s): Stretched Cartesian

- Boundary Conditions: Plunge, pitch; free response (flutter)

- Numerical Algorithm: Relaxation

- Examples:

NACA $64 A 010, M=.72, .80$

$a=00,10$

o Remarks:

Applications of STRANS, UTRANS programs (Traci et al).

Rizzetta, D. P.: A Comparative Study of Two Computational Methods for Calculating Unsteady Transonic Flows About Oscillating Airfoils, AFFDL TR-77-118, November 1977.

o 36 pages, 11 figures, 23 references

- Basic Equations 2-D unsteady small disturbance

- Coordinate System(s): Stretched Cartesian

- No. Mesh Points: $\quad 70 \times 43 ; 99 \times 79$

- Boundary Conditions: Pitch oscillation

- Numerical Algorithm: Relaxation; unsteady ADI

o Examples:

NACA $64 A 010, M=.72, .82, k=.05, .2$

- Remarks:

Compares results of frequency domain method (Traci, et al) with time marching (Ballhaus \& Goorjian) for harmonic motion. 
Rizzetta, D. P.: The Aeroelastic Analys is of a Two-Dimensional Airfoil in Transonic Flow, AFFDL-77-126, December 1977.

- 45 pages, 22 figures, 22 references

- Basic Equations 2-D unsteady small disturbance

- Coordinate System(s): Stretched Cartesian

- No. Mesh Points: $\quad 99 \times 79$

- Boundary Conditions: Pitch, plunge, flap oscillation, free response

- Numerical Algorithm: Unsteady ADI

- Computation Times: 20 minutes/1000 time steps CYBER 74

- Examples: NACA $64 A 010 M=.72, .82$

- Remarks: Uses 3 Degree-of-Freedom equations with ADI method of Ballhaus and Goorjian for flutter analysis by time-integration.

Rose, W. C., and Seginer, A.: Calcuiation of Transonic Flow Over Supercritical Airfoil Sections, AIAA Paper 77-681, AIAA 10th Fluid and Plasma Dynamics Conference, Albequeue, N.W., June 1977.

- 7 pages, 11 figures, 16 references

- Basic Equations

2-D Navier-Stokes

- Coordinate System(s):

Body fitted

o No. Mesh Points:

300

- Boundary Conditions: Exact

- Numerical Algorithm:

Navier Stokes solvers with turbulence model

- Computation Times: 90 minutes on $\operatorname{CDC} 7600$

- Examples: $\quad$ NACA $64 A 010, M=.8$ 
Ruo, S. Y., and Theisen, J. G.: Calculation of Unsteady Transonic Aerodynamics for Oscillating Wings with Thickness, NASA CR-2259, June 1975.

- 51 pages, 12 figures, 46 references

- Basic Equations 3-D unsteady small disturbance, local linearization, harmonic decomposition

- Coordinate System(s): Cartesian

- No. Mesh Points: 25 boxes, 90 boxes

- Boundary Conditions: Linearized

- Examples: 1. Biconvex rectangular wing, $A R=2, \quad \alpha=0.521,0<k<1$ also $\alpha=0$

2. Biconvex delta wing, $A R=1.5$, $0<k<1 \quad \alpha=0,0.05,0.10,0.15$ 3. Bicovex delta wing $A R=1.44$, $0<k<1 \quad \alpha=0.0944$

4. Bicovex delta wing $A R=1.45$, $0<k<1 \quad \alpha=0.06$

5. Parabolic wing, $A R=1.5$, $0<k<1, \alpha=0,0.3$

Schmidt, W., Rohlfs, S., and Vanino, R.: Some Results Using Relaxation Methods for Two- and Three-Dimensional Transonic Flows, 4th International Conference.

- 9 pages, 7 figures, 11 references

- Basic Equations 3-D steady small disturbance

- Coordinate System(s): Cartesian

- No. Mesh Points: $46 \times 12 \times 12$

- Boundary Conditions: Linearized

- Numerical Algorithm: Upwind differencing at hyperbolic points. Standard relaxation.

- Computation Times: Approximately 7 minutes CPU on IBM $370 / 155$

- Examples:

RAE wing $C$ at $M=.95$, ONERA wing $M 6$, PT3 wing body model wing. 
Seebass, A. R., Yu, N. J., and Fung, K-Y: Unsteady Transonic Flow Computations, AGARD Fluid Dynamic Danel Symposium on Unsteady Aerodynamics, September 1977.

- 18 pages, 9 figures, 31 references

- Basic Equations 2 D unsteady time linearized small disturbance

- Coordinate System(s): Coordinates Mapping infinite region into rectangle

o No. Mesh Points: $101 \times 82$

- Boundary Conditions: Linearized

o Numerical Algorithm: ADI with shock fitting for the time dependent equation

- Computation Times: 5 seconds per time step, 60 to 190 time steps/cycle

- Examples:

NACA 64 A006 airfoil, oscillating $1 / 4$ chord flap, and oscillating pitching motion.

o Remarks:

Good comparison of linearized time dependent results with direct solution of nonlinear equation.

South, J. C.: Comments on Difference Schemes for the Three-Dimensional Transonic Small Disturbance Equation for Swept Wings, NASA TMX-71980, July 1976.

- 9 pages, 0 figures, 4 references

- Basic Equations Various forms of 3-D steady small disturbance

o Remarks:

Discusses the various forms of the equation and implications on accuracy and stability. 
Steger, J. L.: Implicit Finite Difference Simulation of Flow About Arbitrary Geometries with Application to Airfoils, AIAA Paper No. 77-665, 10th Fluid and Plasmadynamics Conference, June 1977.

- 14 pages, 12 figures, 32 references

- Basic Equations

2 D unsteady Euler equations, or

"thin layer" Navier-Stokes

o Coordinate System(s): Transformed mapped, body fitted, stretched

0

No. Mesh Points:

$77 \times 27$ - inviscid

$71 \times 33$ - viscous

- Boundary Conditions:

Exact time dependent on time accurate boundary

- Numerical Algorithm: Implicit approximate factorization

- Computation Times: $\quad 0.75 \mathrm{sec} / \mathrm{time}$ step on 7600

- Examples:

1. inviscid NACA 0012,

$M=0.63, .75 \alpha=20$

2. inviscid, linear NACA $64 A 010$,

$M=.8 \alpha=10 \sin \omega t$

3. viscous, linear NACA 0012 ,

$\alpha=00, M=0.2, \operatorname{Re}=104$

4. viscous $18 \%$ 'biconvex, $M=0.75, \alpha$

$=0, \operatorname{Re}=11 \times 106$, turbulent

5. viscous, $18 \%$ biconvex,

$M=0.783, \alpha=0$, buffet case

Traci, R. M., Farr, J. L., and Albano, E.: Perturbation Method for

Transonic Flows About 0scillating Airfoils, AIAA Paper 75-877, AIAA 8th

Fluid and Plasmadynamics Conference, Hartford, Conn., June 1975.

- 12 pages, 14 figures, 22 references

- Basic Equations

2 D Low frequency unsteady time linearized small disturbance equation

- Coordinate System(s): Cartesian, stretched grid

- No. Mesh Points: $50 \times 50$ approx

- Boundary Conditions: Low freq BC's

- Numerical Algorithm: SLOR

- Examples:

NACA 64A006, 64A410 at various $\omega, M$, plunge, pitch + oscillating modes

- Remarks:

Also AIAA J. Vol. 14 No. 9, September 1976. 
Weatherill, W. H., Ehlers, T. E., and Sebastian, J. D.: On the Computation of the Transonic Perturbation Flow Field Around Two- and Three-Dimensional Oscillating Wings, AIAA Paper No. 76-99, 14th Aerospace Sciences Meeting, January 1976.

- 13 pages, 7 figures, 20 references

- Basic Equations $2 D$ and $3 D$ unsteady time linearized small disturbance

- Coordinate System(s): Non-uniform Cartesian

- No. Mesh Points: 2-D: $25 \times 16,34 \times 28,42 \times 30$

3-D: $25 \times 19 \times 20$

- Boundary Conditions: Small disturbance, unsteady; far field doublet

- Numerical Algorithm: SOR

- Computation Times: $7-8 \mathrm{sec}$ per iteration

8-9 sec per for field update

180 iterations for convergence

o Examples:

1. 2-D flat plate, $M=0.875$, pitching oscillation, quasi-steady $(\mathrm{K}=0)$ al so $\mathrm{K}=0.06$

2. $A R=5$ rectangular wing, NACA $64 A 006, M=0.875$, pitching oscillation, $K=0.06, A R=5$.

Weatherill, W. H., Sebastian, J. D., and Ehlers, F. E.: Application of a Finite Difference Method to the Analys is of Transonic Flow Over

Oscillating Airfoils and Winqs, AGARD Conference Proceedings No. 226, Paper No. 17, Unsteady Airloads in Separated and Transonic Flow, 1976.

- 13 pages, 13 figures, 17 references

- Basic Equations

2-D and 3-D small disturbances time linearized

- Coordinate System(s): Cartesian, stretched Cartesian

o No. Mesh Points: 2-D: $28 \times 20,42 \times 30$

3-D: $44 \times 32 \times 26$

- Boundary Conditions: Klunker, outgoing wave, porous wall, free jet, solid wall

- Numerical Algorithm: Row and column relaxation of linear unsteady part 
- Computation Times: 2-D case 30 minutes CDC 6600

- Examples:

2-D - Flat plate oscillating in pitch $M=.9, k=.09, .3$

3-D - Rectangular wing (NACA 64A006 section) $M=.875 \mathrm{k}=.06$ oscillating in pitch

o Remarks:

Basic paper for 3-D frequency domain method. Discusses instability problems for high frequency and possible resolution by direct solution. 


\subsection{Experimental Papers}

Bergh, H., and Tijdeman, H.: Analys is of Pressure Distribution Measured on a Wing With Oscillating Control Surface in Two Dimensional High Subsonic and Transonic Flow, NLR-TR F 253, 1969.

- 32 pages, 38 figures, 25 references

o Facility

- Test Section \&

Wall Conditions:

- $\operatorname{Model}(\mathrm{s}):$

o Mach Numbers:

o Motion Types:

- Frequencies:

o Measurements:

o Remarks:
National Aerospace Laboratory, Netherlands

closed circuit wind tunnel, $\mathrm{h}=.55 \mathrm{~m}, \mathrm{w}=$ $.42 \mathrm{~m}$, longitudinal slotted upper and lower walls

2-D wing, control surface, NACA 65A006

0.5 to 1.02

Sinusoidal oscillation

$30,60,90,120,150 \mathrm{~Hz}$

Detailed measurements of steady and unsteady surface pressures

1. amplitude of oscillation was maximum of 30

2. some discussion on wall interference

Ruhlin, C. L., and Sandford, M. C.: Experimental Parametric Studies of Transonic T-Tail Flutter, NASA TN-D 8066, December 1975.

o 50 pages, 11 figures, 12 references

- Facility: Langley transonic dyramics tunnel

$0 \quad$ Test Section and

Wall Conditions:

o $\quad$ Model (s):

$4.88-\mathrm{m} 2$ test section $=16 \mathrm{ft}$.

slotted walls

$1 / 13$ size wide-body, multijet, cargo/transport with T-tail

a. one tail had load nominal design stiffness for complete model b. one tail had $1 / 2$ nominal design stiffness for only longitudinal tail

- Mach Numbers:

$$
0.7<M<1.0
$$

o Motion Types:

Flutter

- Frequencies:

$0<f<12 \mathrm{H}_{z}$

o Measurements:

Dynamic pressures, flutter frequencies 
Seeamiller, H. L., Marvin, J. G., and Levy, Jr., L. L.: Steady and Unsteady Transonic Flow, AIAA Paper 78-160, 16th Aerospace Sciences Meeting, January 1978.

$$
\begin{aligned}
& \text { - } 14 \text { pages, } 16 \text { figures, } 20 \text { references } \\
& \text { - Facility: NASA Ames high Reynolds No. channel } \\
& \text { - Test Section and } \\
& \text { Wall Conditions: } \\
& \text { - Model(s): } \quad 18 \% \text { circular arc airfoil } \\
& 0 \text { Mach Numbers: } \quad .76, .79 \\
& \text { - Reynolds Numbers: } 11 \times 106 \\
& 0 \text { Motion Types: Steady } \alpha=00 \\
& \text { o. Measurements: Mean velocity, turbulent stress, } \\
& \text { kinetic energy (Laser) surface } \\
& \text { pressures (steady \& unsteady) } \\
& \text { - Remarks: Compares flow field and surface } \\
& \text { pressure fluctuations due to shock } \\
& \text { induced separation with results from } \\
& \text { Navier-Stokes solutions. }
\end{aligned}
$$

Tijdeman, H., Schippers, P., and Persoon, A. J.: Unsteady Airloads on an Oscillating Supercritical Airfoil, AGARD CP-226 Unsteady Airloads in Transonic and Separated Flow, April 1977.
- 15 pages, 27 figures, 23 references
o Facility:
NLR pilot tunnel
0 Test Section and
Wall Conditions:
$.55 \mathrm{~m} \times .42 \mathrm{~m}$, slotted walls
o $\operatorname{Model}(\mathrm{s})$ :
NLR 7301 airfoil
o Mach Numbers:
.5 to 1.0
o Reynolds Number:
$2.1 \times 106$
o Motion Types:
Pitch oscillation
o Frequencies:
$10 \mathrm{~Hz}$ to $80 \mathrm{~Hz}$
o Measurements:
Unsteady pressures, force and moment coefficients
o Remarks:
Compares results with finite
difference calculations 
Tijdeman, H.: Investigations of the Transonic Flow Around Oscillating Airfoils, (PhD Thesis), National Aerospace Laboratory (Netherlands) TR 77090-U, October 1977.

- 146 pages, 0 figures, 188 references

o Facility:

- Test Section and Wall Conditions:

o $\operatorname{Model}(s):$

o Mach Numbers:

o Reynolds Numbers:

o Motion Types:

o Frequencies:

o Measurements:

o Remarks:
NLR pilot tunnel

$.55 \mathrm{~m} \times 42 \mathrm{~m}$, slotted walls

NACA 64A006, NLR 7301 airfoils

.5 to 1.0

$2.1 \times 106$

Oscillations, control surface oscillations

$10 \mathrm{~Hz}$ to $120 \mathrm{~Hz}$

Unsteady \& steady pressures, force and moment coefficients, shock patterns

Compares results with finite difference methods. Reviews status of transonic flow. 
Section III. Summary Chart-Comprehensive Reference List

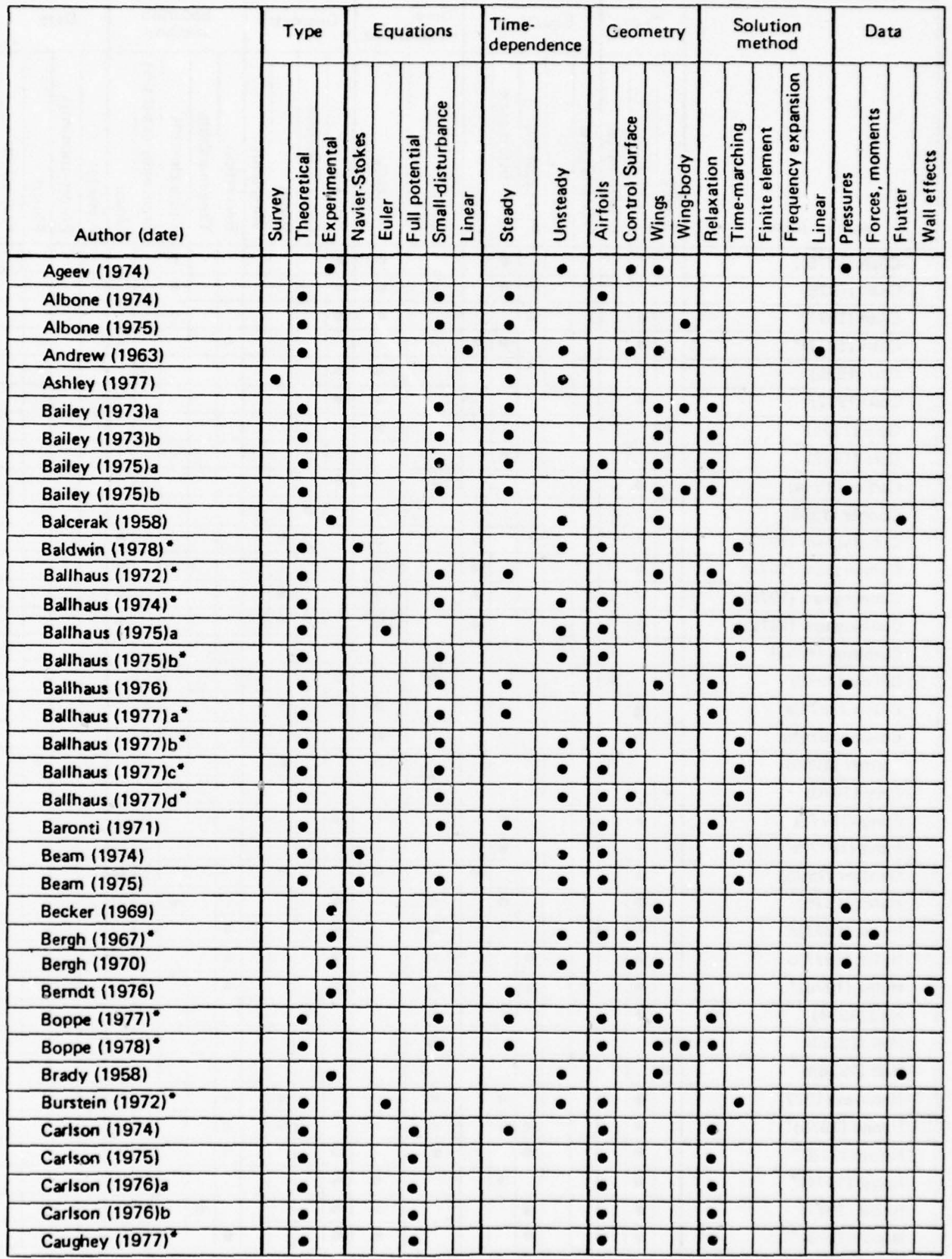

- Reviewed 
Section III. Summary Chart-Comprehensive Reference List (Continued)

\begin{tabular}{|c|c|c|c|c|c|c|c|c|c|c|c|c|c|c|c|c|c|c|c|c|c|}
\hline \multirow[b]{2}{*}{ Author (date) } & \multicolumn{3}{|c|}{ Type } & \multicolumn{5}{|c|}{ Equations } & \multicolumn{2}{|c|}{$\begin{array}{l}\text { Time- } \\
\text { dependence }\end{array}$} & \multicolumn{3}{|c|}{ Geometry } & \multicolumn{4}{|c|}{$\begin{array}{l}\text { Solution } \\
\text { method }\end{array}$} & \multicolumn{4}{|c|}{ Data } \\
\hline & 永 & 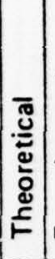 & 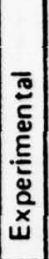 & 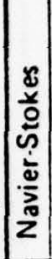 & $\frac{\grave{s}}{\mathrm{~s}}$ & 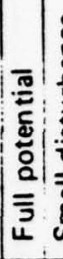 & 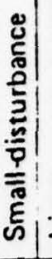 & 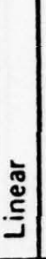 & 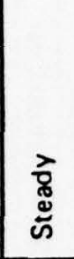 & 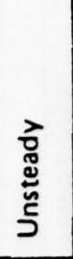 & 高 & 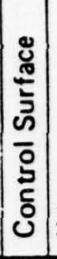 & 年 & 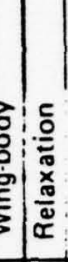 & 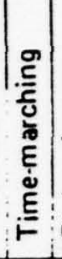 & 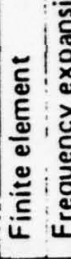 & 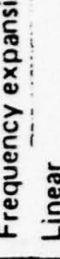 & 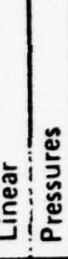 & 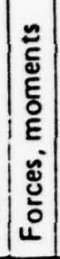 & 产 & 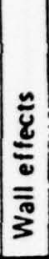 \\
\hline Chan (1975) & & $\bullet$ & & & & & - & & & $\bullet$ & $\bullet$ & & & & & $\bullet$ & & & & & \\
\hline Chan (1976) & & $\bullet$ & & & & & $\bullet$ & & $\bullet$ & $\bullet$ & $\bullet$ & & & & & $\bullet$ & & & & & \\
\hline Chan (1977) & & $\bullet$ & & & & $\bullet$ & & & & $\bullet$ & $\bullet$ & & & & & $\bullet$ & & & & & \\
\hline Chen $(1977)^{\circ}$ & & $\bullet$ & & & & & $\bullet$ & $\bullet$ & $\bullet$ & & & & & $\bullet$ & & & $\bullet$ & $\bullet$ & & & \\
\hline Chu (1976) & & $\bullet$ & & & & & & & & $\bullet$ & $\bullet$ & & & & & & & & & & \\
\hline Chu (1977) & & $\bullet$ & & & & & & & & $\bullet$ & $\bullet$ & & & & & & & & & & \\
\hline Cole (1971) & $\bullet$ & & & & & & & & & $\bullet$ & & & & & & & & & & & \\
\hline Cole (1975) & $\bullet$ & & & & & & $\bullet$ & & & & & & & & & & & & & & \\
\hline Collins (1974) & & $\bullet$ & $\bullet$ & & & & & & $\bullet$ & & $\bullet$ & & & & & & & & & & \\
\hline Cooper (1959), & & & $\bullet$ & & & & & & & $\bullet$ & & $\bullet$ & $\bullet$ & & & & & & & $\bullet$ & \\
\hline Cunningham (1973) & & $\bullet$ & & & & & & $\bullet$ & & $\bullet$ & & & - & & & & $\bullet$ & 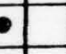 & & & \\
\hline Cunningham (1974) & & $\bullet$ & & & & & & $\bullet$ & & $\bullet$ & & & $\bullet$ & & & & $\bullet$ & - & & & \\
\hline Cunningham (1976)a & & $\bullet$ & & & & & & $\bullet$ & & $\bullet$ & & & $\bullet$ & & & & - & $\bullet$ & & & \\
\hline Cunningham (1976)b & & $\bullet$ & & & & & & - & & $\bullet$ & & & $\bullet$ & & & & $\bullet$ & $\bullet$ & & & \\
\hline Cushman (1953) & & & $\bullet$ & & & & & & & & & & & & & & & & & & $\bullet$ \\
\hline Delery (1976) & & & $\bullet$ & & & & & & $\bullet$ & & $\bullet$ & & & & & & & & & & \\
\hline Ehlers (1974)a & & $\bullet$ & & & & & - & & & $\bullet$ & & & $\bullet$ & & & & $\bullet$ & & & & \\
\hline Ehlers (1974)b & & $\bullet$ & & & & & - & & & $\bullet$ & & & $\bullet$ & & & & $\bullet$ & & & & \\
\hline Farmer (1976) & & & $\bullet$ & & & & & & & $\bullet$ & & & $\bullet$ & & & & & & & $\bullet$ & \\
\hline Frey $(1970)$ & $\bullet$ & & & & & & & & & & & & & & & & & & & & \\
\hline Fung (1977)a & & $\bullet$ & & & & & - & & & $\bullet$ & - & & & & & & $\bullet$ & & & & \\
\hline Fung (1977)b & & $\bullet$ & & & & & - & & & $\bullet$ & $\bullet$ & & & & & & - & & & & \\
\hline Gardner (1976) & & $\bullet$ & & & & & 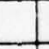 & -1 & & $\bullet$ & & & $\bullet$ & & & & $\bullet$ & 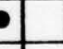 & & & \\
\hline Hafez (1976) & & $\bullet$ & & & & & - & & $\bullet$ & & $\bullet$ & & & & & $\bullet$ & & & & & \\
\hline Hafez (1977)a & & $\bullet$ & & & & & $\bullet$ & & $\bullet$ & & $\bullet$ & & & $\bullet$ & & & & & & & \\
\hline Hafez (1977)b & & $\bullet$ & & & & $\bullet$ & & & $\bullet$ & & $\bullet$ & & & $\bullet$ & & & & & & & \\
\hline Hafez $(1978)^{\circ}$ & & $\bullet$ & & & & $\bullet$ & & & $\bullet$ & & $\bullet$ & & & 0 & & & & & & & \\
\hline Hall (1974) & & $\bullet$ & & & & & $\bullet$ & & $\bullet$ & & & $\bullet$ & & & & & & & & & \\
\hline Hall (1976)a & $\bullet$ & & & & & & & & $\bullet$ & & & & & & & & & & & & \\
\hline Hall (1976)b & $\bullet$ & & & & & & & & & & & & & & & & & & & & \\
\hline Hedman (1977) & & $\bullet$ & & & & & $\bullet$ & & $\bullet$ & & & & - & $\bullet$ & & & & & & & \\
\hline Henne $(1978)^{\circ}$ & & - & & & & $\bullet$ & - & & $\bullet$ & & & & $\bullet$ & - & & & & & & & \\
\hline Holst (1978) & & $\bullet$ & & & & $\bullet$ & & & $\bullet$ & & $\bullet$ & & & - & & & & & & & \\
\hline Isogai $(1974)^{\circ}$ & & $\bullet$ & & & & & $\bullet$ & & & $\bullet$ & $\bullet$ & & & & & & $\bullet$ & & & & \\
\hline Isogai (1977) & & $\bullet$ & & & & $\bullet$ & & & & $\bullet$ & $\bullet$ & & & & $\bullet$ & & & & & & \\
\hline Isogai $(1978)^{\circ}$ & & - & & & & $\bullet$ & & & & $\bullet$ & $\bullet$ & & & $\bullet$ & & & & & & & \\
\hline
\end{tabular}

- Reviewed 
Section III. Summary Chart-Comprehensive Reference List (Continued)

\begin{tabular}{|c|c|c|c|c|c|c|c|c|c|c|c|c|c|c|c|c|c|c|c|c|c|c|c|}
\hline \multirow[b]{2}{*}{ Author (date) } & \multicolumn{3}{|c|}{ Type } & \multicolumn{5}{|c|}{ Equations } & \multicolumn{2}{|c|}{$\begin{array}{l}\text { Time- } \\
\text { dependence }\end{array}$} & \multicolumn{4}{|c|}{ Geometry } & \multicolumn{5}{|c|}{$\begin{array}{l}\text { Solution } \\
\text { method }\end{array}$} & \multicolumn{4}{|c|}{ Data } \\
\hline & 永 & 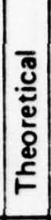 & 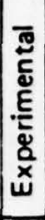 & 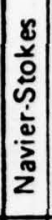 & के & 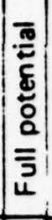 & 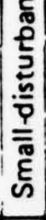 & ¿气 & 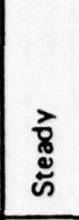 & 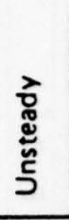 & $\frac{n}{\frac{0}{2}}$ & 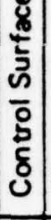 & $\frac{8}{3}$ & $\left|\begin{array}{l}7 \\
8 \\
8 \\
\vdots \\
\frac{c}{3} \\
3\end{array}\right|$ & $\mid$ & 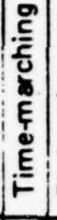 & 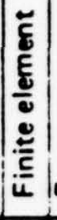 & 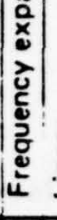 & 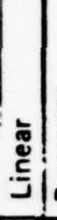 & 这 & 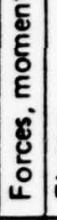 & $\frac{\mathbf{z}}{\frac{2}{u}}$ & 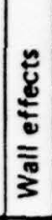 \\
\hline Jameson (1976) & & - & & & & $\bullet$ & & & - & & & & $\bullet$ & & 0 & & & & & & & & \\
\hline Jameson $(1977)^{\circ}$ & & - & & & & $\bullet$ & & & - & & & & $\bullet$ & & - & & & & & & & & \\
\hline Karlsen (1976) & & - & & & - & & & & - & & $\bullet$ & & & & & $\bullet$ & & & & & & & \\
\hline Karisson (1976) & & $\bullet$ & & & & $\bullet$ & & & $\bullet$ & & & & & - & & $\bullet$ & & & & & & & \\
\hline Khosla (1973) & & $\bullet$ & & & & & & & - & & & & & & & & & & & & & & \\
\hline Kimble (1973) & & $\bullet$ & & & & & $\bullet$ & & & - & & & $\bullet$ & & & . & & - & & & & & \\
\hline Kimble (1975) & & $\bullet$ & & & & & $\bullet$ & & & $\bullet$ & & & $\bullet$ & & & . & & $\bullet$ & & & & & \\
\hline Kimble (1976) & & $\bullet$ & & & & $\bullet$ & & & & $\bullet$ & & & $\bullet$ & - & & L & $\bullet$ & & & & & & \\
\hline Klineberg (1976) & & $\bullet$ & & $\bullet$ & & & & & $\bullet$ & & $\bullet$ & & & & & $\bullet$ & & & & & & & \\
\hline Kordulla (1977) & & $\bullet$ & & & & $\bullet$ & & & $\bullet$ & & & & $\bullet$ & & $\bullet$ & & & & & & & & \\
\hline Krupp (1976) & & $\bullet$ & & & & & & & & $\bullet$ & & & & & & 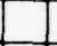 & & & & & & & \\
\hline Landahl (1959)a & & $\bullet$ & & & & & & $\bullet$ & & $\bullet$ & & $\bullet$ & $\bullet$ & & & . & & & $\bullet$ & & & & \\
\hline Landahl (1959)b & & $\bullet$ & & & & & & $\bullet$ & & $\bullet$ & & & $\bullet$ & & & 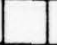 & & & $\bullet$ & & & & \\
\hline Landahl (1962) & & $\bullet$ & & & & & & $\bullet$ & & - & & & & & & 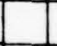 & & & & & & & \\
\hline Landahl (1975) & & $\bullet$ & & & & & & $\bullet$ & & - & & & $\bullet$ & & & 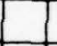 & & & $\bullet$ & & & & \\
\hline Laval (1974) & & $\bullet$ & & & & & & & & & & & & & & - & & & & & & & \\
\hline Laval (1976) & & $\bullet$ & & & $\bullet$ & & & & & $\bullet$ & $\bullet$ & & & & & - & & & & & & & \\
\hline Lee $(1978)^{*}$ & & $\bullet$ & & & & & $\bullet$ & $\bullet$ & $\bullet$ & & $\bullet$ & & $\bullet$ & & $\bullet$ & & $\bullet$ & & $\bullet$ & & & & \\
\hline Lerat (1977) & & $\bullet$ & & & $\bullet$ & & & & & $\bullet$ & - & & & & & 0 & & & & & & & \\
\hline Levy (1977)* & & $\bullet$ & $\bullet$ & $\bullet$ & & & & & & $\bullet$ & & & & & & - & & & & $\bullet$ & & & \\
\hline Loiseau (1967) & & & $\bullet$ & & & & & & & - & & & & & . & . & & & & & & - & \\
\hline Lomax (1971) & & $\bullet$ & & & & & & & - & & - & & & & - & & & & & & & & \\
\hline Lomax (1973)" & & $\bullet$ & & & & & $\bullet$ & & $\bullet$ & & & & $\bullet$ & & - & & & & & & & & \\
\hline Magnus (1974) & & $\bullet$ & & & $\bullet$ & & & & & $\bullet$ & - & & & & & - & & & & & & & \\
\hline Magnus (1975) & & $\bullet$ & & & $\bullet$ & & & & & $\bullet$ & $\bullet$ & & & & & - & & & & & & & \\
\hline Magnus (1976)" & & $\bullet$ & & & $\bullet$ & & & & & - & $\bullet$ & $\bullet$ & & & & - & & & & & & & \\
\hline Magnus (1977)a* & & $\bullet$ & & & $\bullet$ & & & & & - & $\bullet$ & & & & & - & & & & & & & \\
\hline Magnus (1977)b & & $\bullet$ & & & $\bullet$ & & & & & $\bullet$ & $\bullet$ & & & & & - & & & & & & & \\
\hline Magnus (1977)c & & $\bullet$ & & & $\bullet$ & & & & & $\bullet$ & $\bullet$ & $\bullet$ & & & & $\bullet$ & & & & & & & \\
\hline Mason (1978)" & & $\bullet$ & & & & & $\bullet$ & & $\bullet$ & & & & & $\bullet$ & - & & & & & & & & \\
\hline McCroskey (1977) & $\bullet$ & & & & & & & & & $\bullet$ & & & & & & & & & & & & & \\
\hline McCroskey (1978)a & $\bullet$ & & & & & & & & & $\bullet$ & & & & & & & & & & & & & \\
\hline McCroskey $(1978) b^{*}$ & $\bullet$ & & & & & & & & & $\bullet$ & & & & & & & & & & & & & \\
\hline Meier (1974) & $\bullet$ & & & & & & & & & - & & & & & & & & & & & & & \\
\hline Melnik (1973) & & $\bullet$ & $\bullet$ & & & & & & & & & & & & & & & & & & & & $\bullet$ \\
\hline Mirin $(1975)^{\circ}$ & & - & & & $\bullet$ & & & & - & & & & & & & - & & & & & & & \\
\hline
\end{tabular}


Section III. Summary Chart-Comprehensive Reference List (Continued)

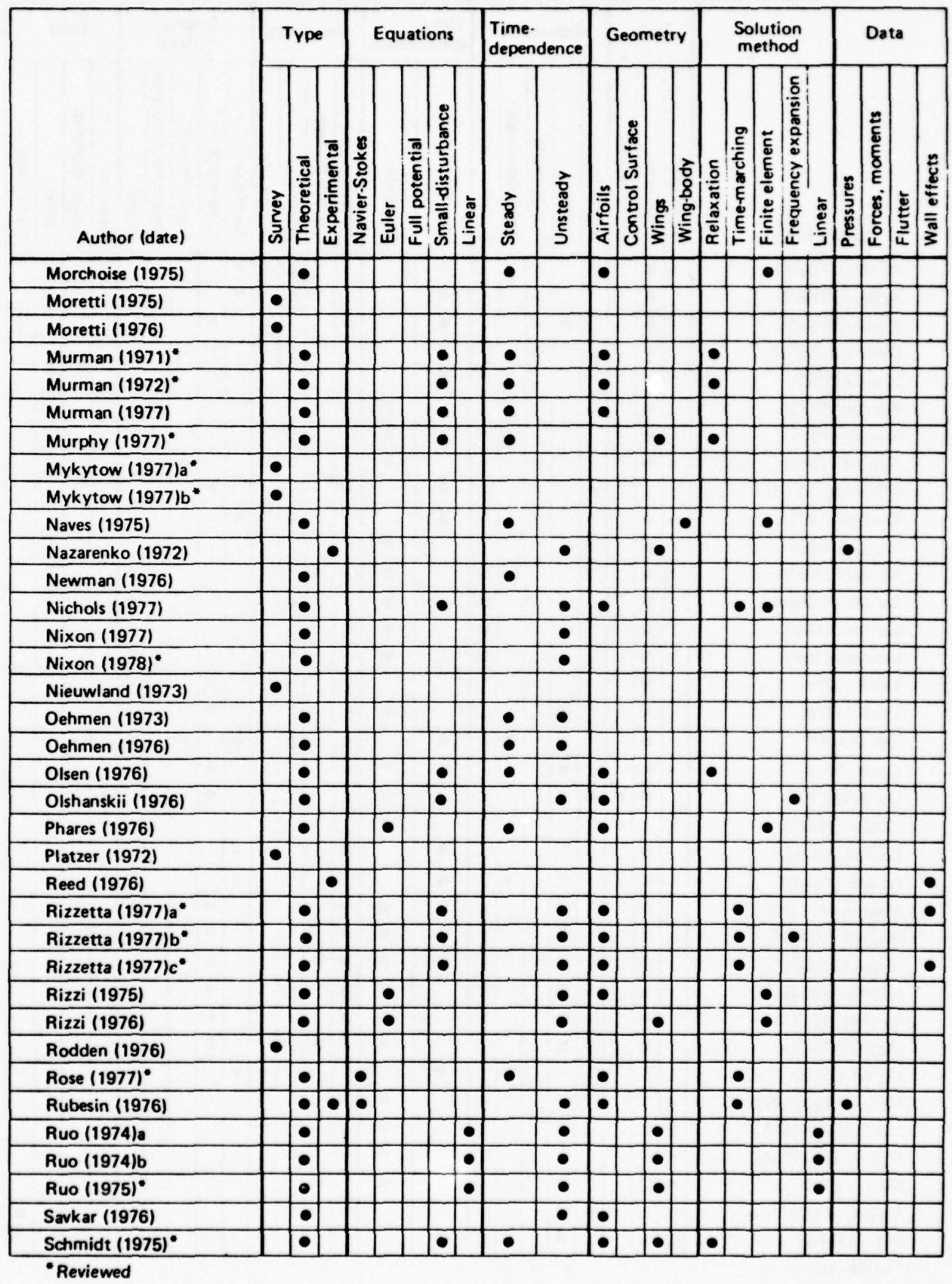


Section III. Summary Chart-Comprehensive Reference List (Continued)

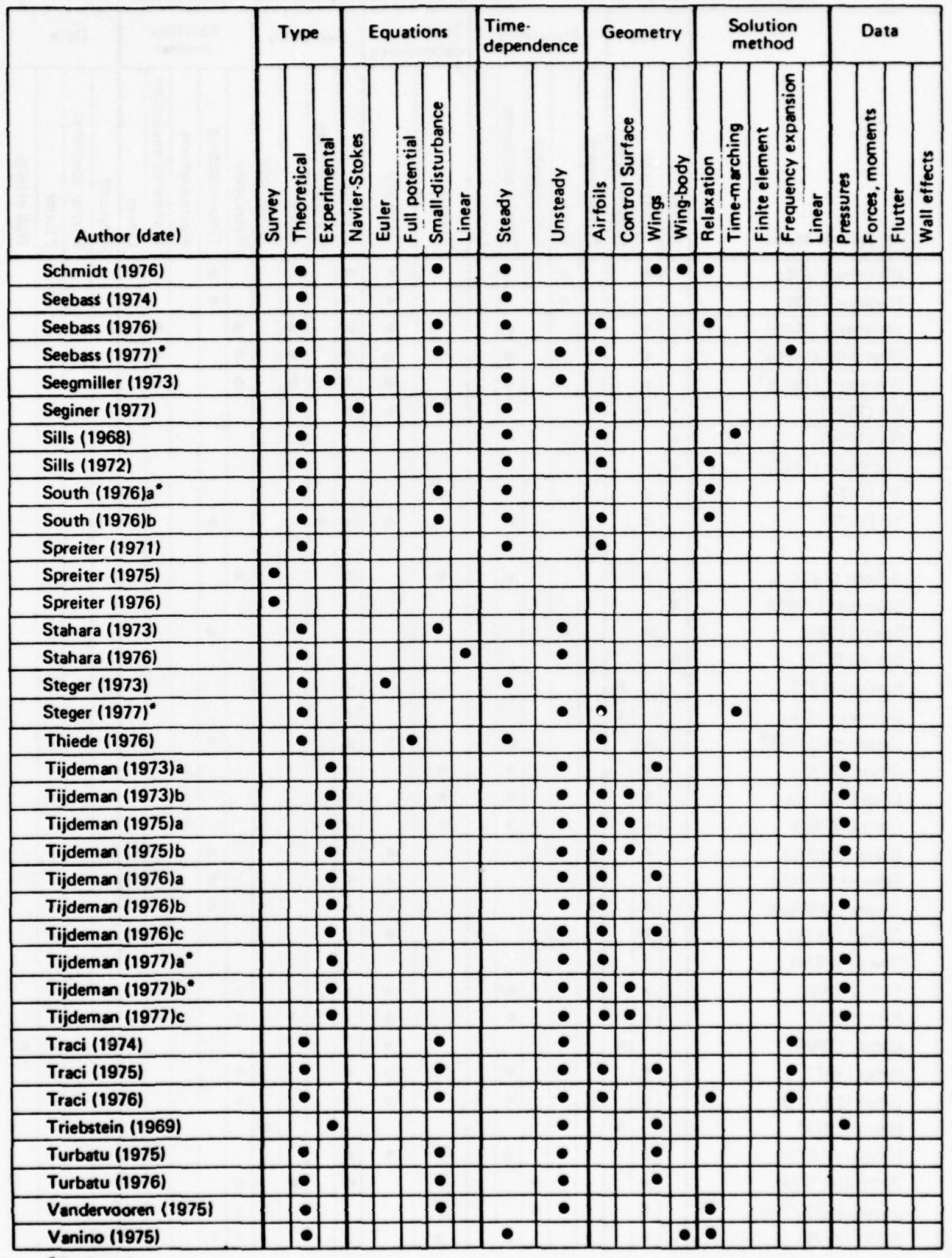

- Reviewed 
Section III. Summary Chart-Comprehensive Reference List (Continued)

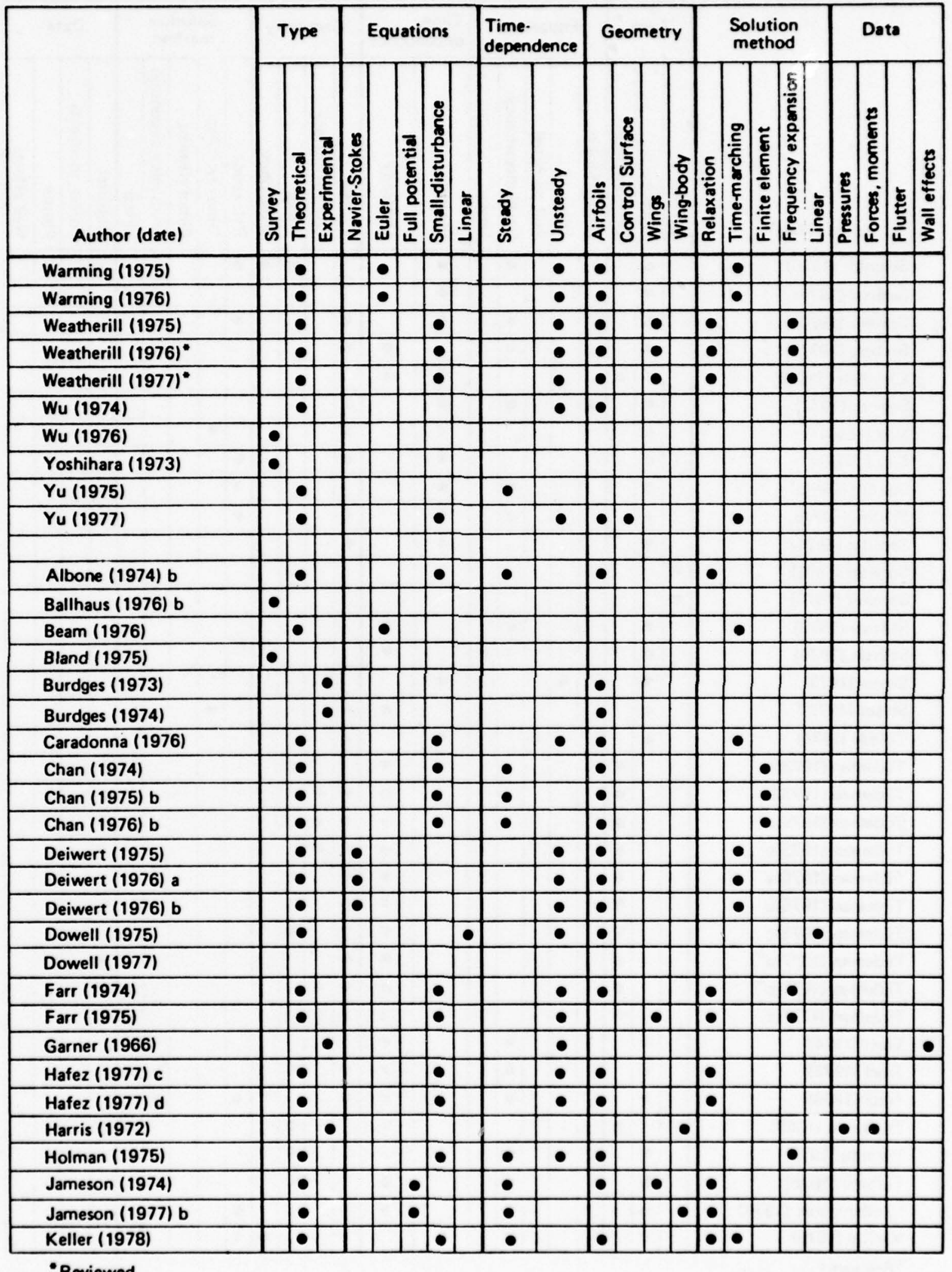




\begin{tabular}{|c|c|c|c|c|c|c|c|c|c|c|c|c|c|c|c|c|c|c|c|c|c|}
\hline \multirow[b]{2}{*}{ Author (date) } & \multicolumn{3}{|c|}{ Type } & \multicolumn{4}{|c|}{ Equations } & \multicolumn{2}{|c|}{$\begin{array}{l}\text { Time- } \\
\text { dependence }\end{array}$} & \multicolumn{4}{|c|}{ Geometry } & \multicolumn{4}{|c|}{$\begin{array}{l}\text { Solution } \\
\text { method }\end{array}$} & \multicolumn{4}{|c|}{ Data } \\
\hline & ڤे & 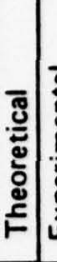 & 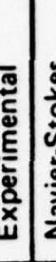 & 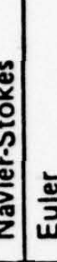 & 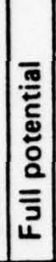 & 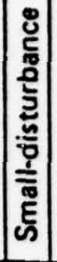 & 高 & $\begin{array}{l}\text { ठे } \\
\text { ळ్ } \\
\text { के }\end{array}$ & 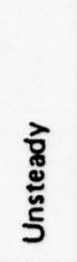 & $\frac{n}{\overline{\frac{n}{2}}}$ & 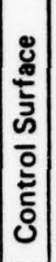 & . & 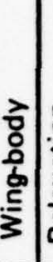 & ) & 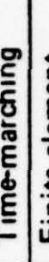 & 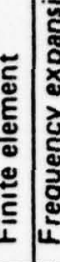 & 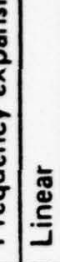 & 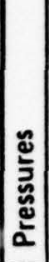 & 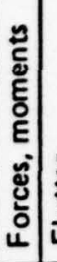 & $\frac{\mathrm{e}}{\mathrm{z}}$ & \\
\hline Klunker (1971) & & $\bullet$ & & & & $\bullet$ & & $\bullet$ & & & & & & . & & & & & & & \\
\hline Krupp (1971) & & $\bullet$ & & & & $\bullet$ & & $\bullet$ & & $\bullet$ & & & & $\bullet$ & & & & & & & \\
\hline Landahl (1961) & $\bullet$ & & & & & - & & & $\bullet$ & & & & & - & & & & & & & \\
\hline Magnus (1970) & & $\bullet$ & & $\bullet$ & & 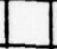 & & $\bullet$ & & $\bullet$ & & & & 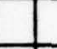 & & & & & & & \\
\hline Malmuth (1976) & & $\bullet$ & 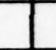 & & & 10 & & - & & $\bullet$ & & 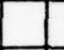 & & - & & & & & & & \\
\hline McDevitt (1976) & & $\bullet$ & $\bullet$ & $\bullet$ & & 7 & & $\bullet$ & & $\bullet$ & & & & 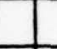 & & & & & & & \\
\hline Murman (1972) & & - & & & & $\bullet$ & & $\bullet$ & & $\bullet$ & & - & & $\bullet$ & & & & & & & \\
\hline Murman (1973) & & $\bullet$ & & & & $\bullet$ & & $\bullet$ & & $\bullet$ & & 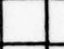 & & -1 & & & & & & & \\
\hline Newman (1971) & - & & & & & & & & & - & & & & 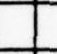 & & & & & & & \\
\hline Nieuland (1967) & & $\bullet$ & & & $\bullet$ & 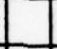 & & $\bullet$ & & $\bullet$ & & - & & & & & & & & & \\
\hline Olsen (1978) & & & & & & 7 & & & & $\bullet$ & $\bullet$ & $\bullet$ & $\bullet$ & 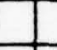 & & & & & & & \\
\hline Shanker (1978) & & $\bullet$ & & & & $\bullet$ & & $\bullet$ & & $\bullet$ & & & & $\bullet$ & & & & & & & 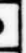 \\
\hline Steger (1973) b & & $\bullet$ & & & & $\bullet$ & & - & & $\bullet$ & & & & & & & & & & & \\
\hline & & & & & & & & & & & & & & & & & & & & & \\
\hline & & & & & & & & & & & & & & & & & & & & & \\
\hline & & & & & & & & & & & & & & & & & & & & & \\
\hline & & & & & & & & & & & & & & & & & & & & & \\
\hline & & & & & & & & & & & & & & & & & & & & & \\
\hline & & & & & & & & & & & & & & & & & & & & & \\
\hline & & & & & & & & & & & & & & & & & & & & & \\
\hline & & & & & & & & & & & & & & & & & & & & & \\
\hline & & & & & & & & & & & & & & & & & & & & & \\
\hline & & & & & & & & & & & & & & & & & & & & & \\
\hline & & & & & & & & & & & & & & & & & & & & & \\
\hline & & & & & & & & & & & & & & & & & & & & & \\
\hline & & & & & & & & & & & & & & & & & & & & & \\
\hline & & & & & & & & & & & & & & & & & & & & & \\
\hline & & & & & & & & & & & & & & & & & & & & & \\
\hline & & & & & & & & & & & & & & & & & & & & & \\
\hline & & & & & & & & & & & & & & & & & & & & & \\
\hline & & & & & & & & & & & & & & & & & & & & & \\
\hline & & & & & & & & & & & & & & & & & & & & & \\
\hline & & & & & & & & & & & & & & & & & & & & & \\
\hline & & & & & & & & & & & & & & & & & & & & & \\
\hline & & & & & & & & & & & & & & & & & & & & & \\
\hline & & & & & & & & & & & & & & & & & & & & & \\
\hline
\end{tabular}


SECTION IV COMPREHENSIVE REFERENCE LIST

AGEEV (1974) Experimental Investigation of The Stable

Self-Oscillations of An Aileron In Transonic Flow;

Ageev, Iu. I. Nazarenko, V. V., Nevezhina, T. P.,

Tsagi, Uchenye Zapiski; Vol. 5, No. 3, 1974, P. 71-80, In Russian, (A76-37929).

ALBONE (1974)a An Improved Numerical Method For Solving The Transonic Small-Perturbation Equation For The Flow Past A Lifting Aerofoil, Royal Aircraft Establishment; Albone, C. M., Catheral1, D., Hall, M. G., Joyce, G., Farnborough (England), August 1974, RAE-TR-74056, (X75-78217).

ALBONE (1974)b A Finite Difference Scheme for Computing Supercritical Flows in Arbitrary Coordinate Systems; Albone, C. M., Farnborough (England), 1974, RAE-TR-74090

ALBONE (1975) Numerical Solutions For Transonic Flows Past Wing-Body Conbinations; Albone, C.M, Hall, M. G., Joyce, G., Royal Aircraft Estabilishment, Farnborough (England), August 1975, RAE-TM-AER0-1645, (X76-73309).

ANDREW (1968) Transonic Unsteady Aerodynamics For Planar Wings With Trailing Edge Control Surfaces; Final Report, Andrew, L. V., Stenton, T. E.; 1 August 1964 - 31 November 1967, Wright-Patterson AFB, Ohio AF, Flight Dyn. Lab., August 1968, AFFDL-TR-67-180, (X69-11330).

ASHLEY (1977) Unsteady Subsonic And Supersonic Inviscid Flow; Ashley, H.; AGARD CP-227, Unsteady Aerodynamics, September 1977.

BAILEY (1973)a Relaxation Methods For Transonic Flow About Wing-Cylinder Combinations And Lifting Swept Wings;

Bailey, F. R., Ballhaus, W. F.; Proceedings of the 3 rd International Conference on Numerical Methods in Fluid Mechanics, 3-7 July 1972, Paris, France, Springer-Verlag, Berlin, Germany, (A74-16951).

BAILEY (1973)b Relaxation Techniques For Three-Dimensional Transonic Flow About Wings; Bailey, F. R., Steger, J. L.; AIAA J. (USA), Vol. II, No. 3, 318-25, March 1973, Also AIAA Paper 72-189, 10th Aerospace Sciences Meeting, January 1972, (A72-16843).

BAILEY (1975)a On The Computation Of Two- And Three-Dimensional Steady Transonic Flows By Relaxation Methods; Bailey, F. R.; Progress in Numerical Fluid Dynamics, 1-77, 1975, Springer-Verlag, Berlin, Germany, (A76-22952). 
BAILEY (1975)

Comparisons of Computed And Experimental Pressures For Transonic Flows About Isolated Wings and Wing Fuselage Configurations; Bailey, F. R., Ballhaus, W. F., National Aeronautics And Space Administration, Ames Research Center, Moffett Field, California, Aerodynamic Analys is Requiring Advanced Computers, Pt. 2 P 1213-1231, (N76-10051).

BALCERAK (1958) Transonic Flutter Model Tests, Part 3 - 60 Degree Delta Wings; Balcerak, J. C., Ostaszewski, N. H.; Wright-Patterson AFB, Ohio, WADC, January 1958 , WADC-TR-56-214, Pt. 3, (X68-82233).

BALDWIN (1978) Thin Layer Approximation And Algebraic Model For Separated Turbulent Flows; Baldwin, B. S., and Lomax, H.; AIAA Paper No. 78-257; 16th Aerospace Sciences Meeting, January 1978.

BALLHAUS (1972) Numerical Calculation Of Transonic Flow About Swept Wings; Ballhaus, W. F., Bailey, F. R.; June 1972, American Institute of Aeronautics and Astronautics, Fluid and Plasma Dynamics Conference, 5th, Boston, Mass., June 26-28, 1972, AIAA Paper 72-677, (A72-34063).

BALLHAUS (1974) The Numerical Simulation of Low Frequency Unsteady Transonic Flow Fields; Ballhaus, W. F., and Lomax, H.; Proceedings of the 4 th International Conference on Numerical Methods in Fluid Dynamics, 57-63, 24-28 June 1974, Boulder, Colo., USA, Springer-Verlag, Berlin, Germany, (A75-42183).

BALLHAUS (1975) Unsteady Transonic Flows Over Airfoils; Ballhaus, W. F., Magnus, R., Yoshihara, H.; Unsteady Aerodyn. Symp, Proc. Univ. of Ariz., Tucson, March 18-20, 1975, Vol.'2, P. 769-791, Publ by Ariz. Board of Regents, Tucson 1975.

BALLHAUS (1975) Implicit Approximate-Factorization Schemes For The Low-Frequency Transonic Equation; Ballhaus, W. F., and Steger, J. L.; NASA TM X-73,082, November 1975.

BALLHAUS (1976) Improved Computational Treatment of Transonic Flow About Swept Wings; Ballhaus, W. F., Bailey, F. R., Frick, J.; National Aeronautics and Space Administration, Advan. in Eng. Sci., Vol. 4, P. 1311-1320, (N77-10348).

BALLHAUS (1976)b Some Recent Progress in Transonic Flow Computations; Ballhaus, W. F., Lecture Series 87 , Computational Fluid Dynamics, von Karman Institute for Fluid Dynamics, March, 1976. 
BALLHAUS (1977)a Implicit Approximate-Factorization Schemes For The Efficient Solution Of Steady Transonic Flow Problems;

Ballhaus, W. F., Jameson, A., Albert, J.; January 1977, NASA-TM-X-73202, (A77-35079, N77-18811).

BALLHAUS (1977)b Implicit Finite-Difference Computations Of Unsteady Transonic Flows About Airfoils, Including The Treatment Of Irregular Shock-Wave Motions; Ballhaus, W. F., Goorjian, P. M.; January 1977, American Institute of Aeronautics and Astronautics, Aerospace Sciences Meeting, 15th Los Angles, California, January 24-26, 1977, AIAA Paper 77-205, (A77-19897).

BALLHAUS (1977)C Efficient Solution Of Unsteady Transonic Flows About Airfoils; Ballhaus, W. F., Goorjian, P. M.; in AGARD CP-226, Unsteady Airloads in Separated And Transonic Flow, April 1977, (N77-31087).

BALLHAUS (1977)d Computation of Unsteady Transonic Flows By The Indicial Method; Ballhaus, W. F., and Goorjian, P. M.; Structures, Structural Dynamics and Materials Conference, 18th, March 21-23, 1977, and Dynamics Specialist Conference, San Diego, California, March 24-25, 1977, Technical Papers, Volume B, New York, American Institute of Aeronautics and Astronautics, Inc., 1977, P. 234-244, AIAA 77-447, also J. AIAA Vol. 10, No. 2, February 1978, (A77-25805).

BALLHAUS (1977)e Unsteady Force And Moment Alleviation In Transonic Flow; Ballhaus, W. F., Goorjian, P. M., and Yoshihara, H.; AGARD CP-227, Unsteady Aerodynamics, September 1977.

BARONTI (1971) Transonic Flows By Coordinate Transformation; Baronti, P. 0., Elzweig, S., Vaglio-Laurin, R.; AIAA J. (USA), Vol. 9, No. 11, 2280, November 1971, (A72-24952).

BEAM (1974) Numerical Calculations of Two Dimensional Unsteady Transonic Flows With Circulation; Beam, R. M., Warming, R. F.; National Aeronautics And Space Administration, Ames Research Center, Moffett Field, California, February 1974, NASA-TN-D-7605, (N74-17021).

BEAM (1975) Numerical Integration of The Small-Disturbance Potential And Euler Equations For Unsteady Transonic Flow; Beam, R. M., Ballhaus, W. F.; National Aeronautics and Space Administration, Ames Research Center, Moffett Field, California, Aerodynamic Analyses Requiring Advanced Computers, SP-347 Pt. 2 P. 789-809, (N76-10036). 
BEAM (1976)

An Implicit Finite Difference Algorithm for Hyperbolic Systems in Conservation--Law Form; Beam, R. M., and Warming, R. F., J. Comp Physics, Vol. 22, No. 1, pp $87-110,1976$.

BECKER (1969) Comparison Of Measured And Calculated Unsteady Pressure Distributions In High Subsonic Flow On An Elastic Swept Wing; Becker, J.; Deutsche Gesellschaft Fuer Luft- und Raumfart, 1969, 32 P. 14 refs. in German, DGLR-69-31, (A70-15151, N70-24944).

BERGH (1967) Analysis of Pressure Distributions Measured On A Wing With Oscillating Control Surface In Two-Dimensional High Subsonic And Transonic Flow; Bergh, H., Tijdeman, H.; National Lucht-en

Ruimetevaart laboratorium, Amsterdam, Netherlands, March 1967, NLR-TR-F-253, (N69-11754).

BERGH (1970) High Subsonic And Transonic Effects On Pressure Distributions Measured For A Swept Wing With Oscillating Control Surface; Bergh, H., Tijdeman, H., Zwaan, R. J.; Zeitschrift Fuer Flugwissenschaften, Vol. 18, P. 339-347, September 1970, (A70-44673).

BERNDT (1976) Transonic Flow At A Slotted Test Section Wall Final Report; 1 October 1971 - 30 September 1976, Aeronautical Research Inst. of Sweden, Stockholm, AF0SR-77-0035TR, (N77-25194).

BLAND (1975) Recent Advances and Concepts in Unsteady Aerodynamic Theory; Bland, S. R., National Aeronautics and Space Administration, Ames Research Center, Moffet Field, Californis, Aerodynamic Analys is Requiring Advanced Computers, SP-347, Pt 2, pp 1305-1326.

BOPPE (1977) Calculation Of Transonic Wing Flows By Grid Embedding; Boppe, C. W.; January 1977, American Institute of Aeronautics and Astronautics, Aerospace Sciences Meeting, 15th, Los Angles, California, January 24-26, 1977, AIAA Paper 77-207, (A77-19899).

BOPPE (1978) Computational Transonic Flow About Realistic Aircraft Configurations; Boppe, C. W.; AIAA 16th Aerospace Science Meeting, January 16-18 1978, AIAA Paper 78-104.

BRADY (1958) Transonic Flutter Model Tests, Part 2 - Straight Wings; Brady, W. G., King, S. R., Maier, H. G.; Wright-Patterson AFB, Ohio, WADC, January 1958, WADC-TR-56-214, Pt. 2, (X68-82192).

BURDGES (1973) A Synthesis of Transonic 2-D Airfoil Technology, AIAA Paper 73-792, 1973. 
BURDGES (1974) High Reynolds Number Test of a NASA 10-Percent-Thick Supercritical Airfoil Section at Transonic Speeds, NASA CR-132468, 1974.

BURSTEIN (1972) Time Dependent Calculations For Transonic Flow;

Burstein, S. Z., Mirin, A. A.; Proceedings of the 3 rd

International Conference on Numerical Methods in Fluid

Mechanics, II, 1973, 3-7 July 1972, Paris, France,

Springer-Verlag, Berlin, Germany.

CARADONNA (1976) Numerical Calculation of Unsteady Transonic Potential Flow over Helicopter Rotor Blades; Carradonna, F. X., and Ison, M. P., J. AIAA Vol. 14, No. 4, pp 482-488, April 1976.

CARLSON (1974) Inverse Transonic Flow Calculations Using Experimental Pressure Distributions; Carlson, L. A; AIAA J. (USA), Vol. 12, No. 4, 571-2, April 1974, (A74-28050).

CARLSON (1975) Transonic Airfoil Flowfield Anaiysis Using Cartesian Coordinates, Final Report; Carlson, L. A.; Texas A\&M Univ., August 1975, NASA-CR-2577.

CARLSON (1976)a Inverse Transonic Airfoil Design Including Viscous Interaction; Carlson, Leland A.; Soc of Eng Sci Annu Meet, 13th, Hampton, Va., Nov 1-3, 1976, (N77-10354).

CARLSON (1976)b Transonic Airfoil Analysis And Design Using Cartesian Coordinates; Carlson, Leland A.; J. Aircr V-13 n 5 May 1976 P. 349-356.

CAUGHEY (1977) Numerical Calculation of Transonic Potential Flow About Wing-Fuselage Combinations; Caughey, D. A., and Jameson, A.; June 1977, American Institute of Aeronautics and Astronautics, Fluid and Plasma Dynamics Conference, 10th, Albuquerque, N. Mex., June 27-29, 1977, AIAA Paper 77-677, (A77-37028).

CHAN (1974) Finite Element Analysis of Transonic Flow, Chan, S. T. K. and Brashears, M. R., AFFDL TR-74-11, March, 1974.

CHAN (1975)a Finite Element Analysis Of Unsteady Transonic Flow; Chan, S. T. K., Brashears, M. R.; June 1975, American Institute of Aeronautics and Astronautics, Fluid and Plasma Dynamics Conference, 8th, Hartford, Conn., June 16-18, 1975, AIAA Paper 75-875, (A75-33960).

CHAN (1975)b Computer Program for Steady Transonic Flow over Thin Airfoils by Finite Elements; Chan, S.T. K., and Brashears, M. R., AFFDL TR-75-126, October, 1975. 
CHAN (1976)a

CHAN (1976)b

CHAN (1977)

CHEN (1977)

$\mathrm{CHU}(1976) \mathrm{a}$

CHU (1976)b

COLE (1971)

COLE (1975)

COLLINS (1974)
Analysis Of Transonic Flow Over Lifting And Oscillating Airfoils; Chan, S. T. K., Brashears, M. R.;

International Symposium on Finite Element Methods in Flow Problems, 2nd, Santa Margherita, Ligure, Italy, June 14-18, 1976, International Centre for Computer Aided Design, 1876, P. 219-230, (A77-47542).

Finite Element Analysis of Transonic Flows over Thin Airfoils, Volume I; Chan, S.T.K., Chen, H. C., and Brashears, M. R., AFFDL-76-49, Volume I, May, 1976.

Finite Element Applications To Unsteady Transonic Flow; Chan, S. T. K, and Chen, H. C.; Structures, Structurdl' Dynamics and Materials Conference, 18th, March 21-23, 1977, and Dynamics Specialist Conference, San Diego, Calif., March 24, 25, 1977, Technical Papers, Volume B, New York, American Institute of Aeronautics and Astronautics, Inc., 1977, P. 225-233, A1AA 77-446, (A77-25804).

A Far Field Matching Method For Transonic Computation; Chen, A. W., Dickson, L. J., and Rubbert, P. E.; AIAA Paper 77-208, 15th Aerospace Sciences Meeting, January 1977.

Unsteady Two-Dimensional Subsonic, Transonic And Supersonic Flow Calculations By The Method of Characteristics; Chu, C. W., and Nadir, S.; Northrop Corp., (NDR 76-19), February 1976, (N76-29168).

Unsteady Two-Dimensional Subsonic, Transonic, And Supersonic Flow Calculations By The Method of Characteristics; Chong-Wei Chu, Nadir, S.; Proceedings of the 5 th International Conference on Numerical Methods in Fluid Dynamics, 145-52 1976, 28 June - 3 July 1976, Enschede, Netherlands, Springer-Verlag, Berlin, Germany.

Computational Techniques For Unsteady Transonic Flow; Cole, J. D., Krupp, J. A.; Cal ifornia Univ., Los Angeles, School of Engineering and Applied Science, 71, NASA-CR-136998, (X74-71970).

Modern Developments In Transonic Fiow; Cole, J. D., Siam J. Appl. Math., Vol. 29, No. 4, 763-87, December 1975.

Experimental And Theoretical Investigations In Two-Dimensional Transonic Flow; Collins, Donald J., Krupp, James A.; AIAA J Vol. 12 No. 6 June 1974, P. 771-778. 
COOPER (1959) Transonic And Supersonic Flutter Model Tests of Low Aspect Ratio Unswept All-Movable Stabilizers;

Cooper, R. E., Kazmerzak, L. E.; Wright-Patterson AFB, Ohio, WADC August 1959, WADC-TR-59-406, (X68-82103).

CUNNINGHAM (1973) The Application of General Aerodynamic Lifting Surface Eiements To Problems In Unsteady Transonic Flow;

Cunningham, A. M., Jr., General Dynamics/Fort Worth, Tex., Convair Aerospace Div., February 1973, NASA-CR-112264, (N73-21272).

CUNNINGHAM (1974) An 0scillatory Kernel Function Method For Lifting Surfaces In Mixed Transonic Flow; Cunningham, A. M. Jr.; American Inst. Aeronautics and Astronautics, ASME, Soc. Automotive Engrs, AIAA/ASME/SAE 15th Structures, Structural Dynamics and Materials Conference, 17-19 April 1974, (A74-26669).

CUNNINGHAM (1976) The Calculation of Steady Non-Linear Transonic Flow a Over Finite Wings With Linear Theory Aerodynamics; Cunningham, A. M. Jr., General Dynamics/Fort Worth, Tex., August 1976, NASA-CR-145023, (N76-28194).

CUNNINGHAM (1976) A Steady And Oscillatory Kernel Function Method For b Interfering Surfaces In Subsonic, Transonic And Supersonic Flow; Cunningham, A. M., Jr., General Dynamics/Fort Worth, Tex, September 1976, NASA-CR-144895, (N76-33138).

CUSHMAN (1953) Small Scale Transonic Interference Studies With Perforated Wall Test Sections; Cushman, H. T.; United Aircraft Corp., East Hartford, Conn., June 1953, AF 18/600/-171, (x68-82166).

DEIWERT (1975) Numerical Simulation of High Reynolds Number Transonic Flows; Deiwert, G. S., J. AIAA, Vol. 13, No. 9, pp 1354-1359, October, 1975 .

DEIWERT (1976)a Computation of Separated Transonic Turbulent Flows, Deiwert, G. S., J. AIAA, Vol. 14, No. 6, pp 735-740, June, 1976.

DEIWERT (1976)b Recent Computation of Viscous Effects in Transonic Flow; Deiwert, G.S., Proceedings of the 5 th International Conference on Numerical Methods in Fluid Dynamics 159-64. 197628 June - 3 July 1976, Euschede, Netherlands, Springer-Verlag, Berlin, Germany

DELERY (1976) Viscous Interaction With Separation In Transonic Flow; Chattot, J. J., Leballeur, J. C.; August 1976, Trans1. into English of Onera Conf. Paper presented at Flow Separation, AGARD Fluid Dyn. Symp., Goettingen, West Germany, 27-30 May 1975, NASA-TT-F-17187, (N77-10997). 
DOWELL (1975) Simplified Theory of Oscillating Airfoils in Transonic Flow; Dowe11, E. H., Proceedings of Symposium on Unsteady Aerodynamics, Vol. 2, pp 655-679, University of Arizona, Tuscon, March 19-20, 1975.

DOWELL (1977) A Simplified Theory of Oscillating Airfoils in Transonic Flow--Review and Extension; Dowell, E. H., AIAA Dynamics Specialists Conference, March, 1977.

EHLERS (1974)a A Finite Difference Method For The Solution of The Transonic Flow Around Harmonically Oscillating Wings; Ehlers, F. E.; June 1974, American Institute of Aeronautics and Astronautics, Fluid and Plasma Dynamics Conference, 7th, Palo Alto, Cal if., June 17-19, 1974, AIAA Paper 74-543, (A74-33138).

EHLERS (1974)b A Finite Difference Method For The Solution of The Transonic Flow Around Harmonically Oscillating Wings; Ehlers, E. F.; Boeing Commercial Airplane Co., Seattle, Washington, July 1974, NASA-CR-2257.

FARMER (1976) Comparison of Supercritical And Conventional Wing Flutter Characteristics; Farmer, M. G., Hanson, P. W., Wynne, E. C.; National Aeronautics and Space Administration, Langley Research Center, Langley Station, Va., May 1976, presented at the AIAA/ASME/SAE 17 th Struct., Structural Dyn., and Mater. Conf., Valley Forge, Pa., 5-7 May 1976, NASA-TM-X-72837.

FARR (1974) Computer Programs for Calculating Small Disturbance Transonic Flows About Oscillating Airfoils, AFFDL TR-74-135, November, 1974.

FARR (1975) Computer Programs for Caclulating Small Disturbance Transonic Flows About Oscillating Planar Wings; AFFDL-75-101, 1975

FREY (1970) Calculative Techniques For Transonic Flows; Frey, W. H., Spreiter, J. R.; Stahara, S. S., NASA, Anal. Methods In Aircraft Aerodyn, 1970.

FUNG (1977)a Small Unsteady Perturbations In Transonic Flows; Fung, K-Y, Yu, N. J., Seebass, R.; Arizona Univ Tucson Dept. of Aerospace and Mechanical Engineering, GRA17804, November 1977.

FUNG (1977)b Small Unsteady Perturbation In Transonic Flows;

Fung, K. Y., Yu, N. J., and Seebass, R.; AIAA Paper 77-675, 10th Fluid and Plasmadynamics Conference, June 1977. 
GARNER (1966) Subsonic Wind Tunnerl Wall Correct ions, Garner, A. C., Rogers, E.W.E., Acum, W.E.A., and Maskell, E. C., AGARDograph 109, October, 1966.

GARNER (1976) A Practical Approach To The Prediction Of Oscillatory Pressure Distributions On Wings In Supercritical Flow; Garner, H. C.; Royal Aircraft Establishment, Farnborough (England), RAE-TR-74181, (N77-19017).

HAFEZ (1976) Application Of Finite Element Approach To Transonic Flow Problems; Hafez, M. M., Murman, E. M., Wellford, L. C.; Nasa, Langley Res. Center Advan. in Eng., Sci., Vol. 4, P. 1371-1393, (N77-10353).

HAFEZ (1977)a Shock-Fitting Applied To Relaxation Solutions of Transonic Small-Disturbance Equations; Hafez, Mohammed M., Cheng, H. K.; AIAA J Vol. 15 No., 6 June 1977 , P. $786-793$.

HAFEZ (1977)b Numerical Solution of the Unsteady Transonic Small Disturbance Equations; Hafez, M. M., Rizk, M. H., and Murman, E. M.; AGARD CP-226, Unsteady Airloads in Separated and Transonic Flow, April, 1977.

HAFEZ (1977)c A Shock-Fitting Algorithm For The Full Potential Equation; Hafez, M. M., Murman, E. M.; Computational FLuid Dynamics Conierence, 3rd, Albuquerque, N. Mex., June 27, 28, 1977, New York, American Institute of Aeronautics and Astronautics, Inc., 1977, P. 2-12, AIAA 77-632, (A77-35077).

HAFEZ (1977)d Numerical Solution of the Unsteady Transonic Small-Disturbance Equations; Hafez, M. M., Rizk, M. H., Murman, E. M., and Wellford, L. C.; AFFDL-TR-77,100.

HAFEZ (1978) Artificial Compressibility Methods For Numerical Solution of Transonic Full Potential Equation; Hafex, M. M.; AIAA 11th Fluid \& Plasmadynamics Conference, July 1978, AIAA Paper 78-1148.

HALL (1974) Recent Developments In Methods For Calculating Transonic Flows Over Wings; Hall, M. G., Firmin, M. C. P.; Int Conc of the Aeronaut Sci (ICAS), 9th Congr. Proc. Haifa. Isr. Aug. 25-30, 1974 Vol. 1, Fluid Dyn. Aerodyn and Gas Dyn P. 134-151, publ by Weizmann Sci Press of Isr. Jerusalem, 1974.

HALL (1976)a Methods And Problems In The Calculation Of Transonic Flows; Hall, M. G.; Proceedings of the 5 th International Conference on Numerical Methods in Fluid Dynamics, 65-74, 1976, 28 June - 3 July 1976, Enschede, Netherlands, Springer-Verlag, Berlin, Germany, (A77-28366). 
HALL (1976)

Transonic Flows; Hall, M. G.; Computational Methods and Problems in Aeronautical Fluid Dynamics, London and New York, Academic Press, 1976, p. 242-269, (A77-12561).

\section{HARRIS (1972) \\ Wing Tunnel Measurements on a NASA Supercritical Wing Research Airplane Configuration, NASA TMS-2469,} February 1972.

HEDMAN (1977) Pressure Distributions For A Swept Wing Body Configuration Obtained From Coupling Transonic Potential Flow Calculations And Boundary Layer Calculations; Hedman, S. G.; AGARD Prediction of Aerodynamic Loading, February 1977, (N77-20006).

HENNE (1978) Transonic Wing Analysis Using Advanced Computational Methods; Henne, P. A., Hicks, R. M.; AIAA Paper 78-105, 16 th Aerospace Sciences, January 1978.

HOLMAN (1975) A User's Guide for the Improved Versions of the Computer Programs STRANS and UTRANS; Holman, W. L., AFFDL-TM-75-168=FYS, December, 1975.

HOLST (1978) An Implicit Algorithm For The Conservative Transonic Full Potential Equation Using An Arbitrary Mesh; Holst, T. L.; AIAA Paper 78-1113, 11th Fluid and Plasmadynamics Conference, July 1978.

ISOGAI (1974) Unsteady Transonic Flow Over Oscillating Circular-Arc Airfoils; Isogai, K.; April 1974, AIAA, ASME, and SAE, Structures, Structural Dynamics and Materials Conference, 15th, Las Vegas, Nev., April 17-19, 1974, AIAA Paper $74-360$, (A74-26670).

ISOGAI (1977) Calculation Of Unsteady Transonic Flow Over Oscillating Airfoils Using The Full Potential Equation; Isogai, K.; Structures Dynamics and Materials Conference, 18th, March 21-23, 1977, and Dynamics Specialist Conference, San Diego, Calif., March 24, 25, 1977, Technical Papers, Volume B, New York, American Institute of Aeronautics and Astronautics, Inc., 1977, P. 245-256, AIAA 77-448, (A77-25806).

JAMESON (1974) Iterative Solution of Transonic Flows over Airfoils and Wings, including Flows at March 1 ; Jameson, A. Comm. of Pure and App. Math, Vol. 27, pp 283-309, May 1974 (A74-43451).

JAMESON (1975) Transonic Potential Flow Calculations Using Conservation Form; Jameson, A.; Proceedings of Second AIAA Conference on Computational Fluid Dynamics, Hartford, Conn. pp 148-161, June, 1975.

ISOGAI (1978) Numerical Study of Transonic Flow Over Oscillating Airfoils Using The Full Potential Equation; Isogai, K.; National Aeronautics and Space Administration, Langley Research Center, NASA TP 1120, April 1978. 
JAMESON (1976) A Brief Description of The Jameson-Caughey NYU Transonic Swept-Wing Computer Program, FL022 Jameson, A., Caughey, D. A., Newman, P. A., Davis, R; Nationai Aeronautics and Space Administration, Langley Research Center, Langley Station, Va., NASA-TM-X-73996, (N77-15977).

JAMESON (1977)a Numerical Calculation of The Transonic Flow Past A Swept Wing; Jameson, A., Caughey, D. A.; New York Univ., N. Y. ERDA Mathematics and Computing Lab., June 1977, NASA-CR-153297, (N77-27071).

JAMESON (1977)b A Finite Volume Method for Transonic Potential Flow Calculations; Jameson, A., and Caughey, D. A., AIAA Paper 77-635, 10th Fluid and Plasmadynamics Conference, Albuquerque, New Mexico, June 27-29, 1977.

KARLSEN (1976) A Consistent Finite-Difference Operator For Time-Dependent Solution of Steady, Oblique Shocks; Karlsen, L. K.; Royal Inst. of Tech., Stockholm (Sweden), KTH-AERO-FI-47, (N77-12331).

KARLSSON (1976) The Method of Decomposition Applied In Transonic Flow Calculations; Karlsson, K. R., Sedin, Y. C.-J; Proceedings of the 5 th International Conference on Numerical Methods in Fluid Dynamics, 262-7, 1976, 28 June -. 3 July 1976, Enschede, Netherlands, Springer-Verlag, Berlin, Germany, (A77-28391).

KELLER (1978( Preliminary Study of the Use of the STAR-100 Computer for Transonic Flow Calculations; Keller, J. D., and Jameson, A., AIAA Paper 78-12, 16th Aerospace Sciences Meeting, Huntsville, Alabama, Jan. 16-18, 1978.

KHOSLA (1973) Transonic Flow Calculated In Two- And Three-Dimensions; Khosla, P. K., Rubin, S. G.; Polytech Inst Brooklyn, Dep Aerospace Eng Appl Mech, PIBAL Rep n 73-13, June 1973.

KIMBLE (1973) Unsteady Transonic Flow Analysis For Low Aspect Ratio, Pointed Wings; Kimble, K. R., Ruo, S. Y., Wu, J. M., Liu, D. Y.; January 1973, American Institute of Aeronautics and Astronautics Aerospace Sciences Meeting, 11th, Washington, D. C., January 10-12, 1973, AIAA Paper 73-122, (A73-16878).

KIMBLE (1975) Numerical Calculations of Unsteady Forces Over Thin Pointed Wings In Sonic Flow; Kimble, K. R., Wu, J. M.; Unsteady Aerodyn. Symp. Proc. Univ of Ariz. Tucson, March 18-20, 1975 Vol. 2 P. 583-607, Publ by Ariz Board of Regents, Tucson, 1975. 
KIMBLE (1976) A Finite Element Solution Of Unsteady Transonic Flow Problems For Three-Dimensional Wings And Bodies;

Kimble, K. R.; July 1976, American Institute of

Aeronautics and Astronautics, Fluid and Plasma Dynamics Conference, 9th, San Diego, Cal if., July 14-16, 1976, AIAA Paper 76-328, (A76-36987).

KLINEBERG (1976) Calculation of Separated Flows At Subsonic And

Transonic Speeds; Klineberg, J. M., and Steger, J. L.; Proceedings of 5 th International Conference on Numerical Methods in Fluid Dynamics, 28 June - 3 July 1976, Enschede, Netherlands, Springer-Verlag, Berlin, Germany.

KLUNKER (1971) Contributions to Methods for Calculating the Flow About Thin Lifting Wings at Transonic Speeds--Analytical Expression for the Far Field, Klunker, E. B., NASA TND-6530, 1971.

KORDULLA (1977) Investigations Related To The Inviscid-Viscous Interaction In Transonic Flows About Finite 3-D Wings; Kordulla, W.; January 1977, American Institute of Aeronautics and Astronautics, Aerospace Sciences Meetings, 15th, Los Angeles, Cal if., Jan. 24-26, 1977, 14 p., AIAA Paper 77-209, (A77-22244).

KRUPP (1971) Computation of Transonic Flows Past Lifting Airfoils and Slender Bodies; Krupp, J. A., and Murman, E. M.; AIAA 4th Fluid and Plasma Dynamics Conference, Palo Alto, California, June 21-23, 1971.

KRUPP (1976) Studies In Transonic Flow 4: Unsteady Transonic Flow Final Report; Krupp, J. A., Cole, J. D.; California Univ., Los Angeles, School of Engineering and Applied Science, NASA-CR-149042, (N77-70922).

LANDAHL (1959) Theoretical Studies of Unsteady Transonic Flow. Part 4 - The Oscillating Rectangular Wing With Control Surface; Landah1, M. T.; Aeronautical Research Inst. of Sweden, Stockholm, 1959, FFA-80, (N68-86806).

LANDAHL (1959) Theoretical Studies of Unsteady Transonic Flow. Part 5 - Solution For The Delta Wing And Wings of General Polygonal Planforms; Landahl, M. T.; Aeronautical Research Inst. of Sweden, Stockholm, January 1959, FFA-81, (N68-87805).

LANDAHL (1961) Unsteady Transonic Flow; Landahl, M., Pergamon Press, New York, 1961.

LANDAHL (1962) Linearized Theory For Unsteady Transonic Flow, Landahl, M. T., International Union of Theoretical and Applied Mechanics; Symposium Transsonicum, Aachen, West Germany, September $3-7,1962$, Berlin Springer-Verlag, 1964, P. 414-439, (A65-12347). 
LANDAHL (1975)

LAVAL (1974)

LAVAL (1976)

LEE (1978)

LERAT (1977)

LEVY (1977)

LOISEAU (1967)

LOMAX (1971)

LOMAX (1973)
Some Developments In Unsteady Transonic Flow Research; Landah I, M. T.; Symposium Transsonicum II, Goettingen, West Germany, September 8-13, 1975, Proceedings,

Berl in, Springer-Verlag, 1976, P. 1-32, (A76-27827).

Time-Dependent Methods For Computing Wall Effects In Two-Dimensional Supercritical Flow; Laval P.; European Space Research Organization, Paris, Aerospace Res. (ESRO-TT-40), April 1974, (N75-10369).

Calculation of Nonstationary Transonic Flow Around An Oscillating Profile By A Fractional Step Method;

Laval, P.; 12th Biennial Fluid Dynamics Symposium on Advanced Problems and Methods in Fluid Dynamics, 8-13 Septebmer 1975, Poland, French, (A77-051079).

An Improved Matching Method For Transonic Computations; Lee, K. D., Dickson, L. J., Chen, A. W., and Rubbert, P. E.; AIAA Paper 78-1116, 11th Fluid and Plasmdynamics Conference, July 1978.

Numerical Calculation of Unsteady Transonic Flows; Lerat, A., Sides, J.; Agard Unsteady Airloads in Separated and Transonic Flow, 10 p, April 1977, CP 226, (A77-44955).

An Experimental And Computational Investigation of The Steady And Unsteady Transonic Flow Fields About An Airfoil In A Solid-Wall Test Channel; Levy, L. L., Jr.; June 1977, American Institute of Aeronautics and Astronautics, Fluid and Plasmadynamics Conference, 10 th, Albuquerque, N. Mex., June 27-29, 1977, AIAA Paper 77-678, (A77-37029).

Experimental Study of Single-Degree of Freedom Flutter In Transonic Flow; Loiseau, H.; February 1967, Transl. into English from office Natl., D'Etudes Et De Rech, Aerospatiales Report Tn-95, NASA-TT-F-10729, (N67-25019).

Numerical Calculation of Transonic Flow About Two-Dimensional Airfoils By Relaxation Procedures; Lomax, H., Steger, J. L.; American Institute of Aeronautics and Astronautics, Fluid and Plasma Dynamics Conference, 4th, Palo Alto, Calif., June 21-23, 1971, AIAA Paper 71-569, (A71-31562).

On The Numerical Simulation of Three-Dimensional Transonic Flow with Application To The C-141 Wing; Lomax, H., Bailey, F. R., and Ballhaus, W. F.; NASA TN $D-6933$, August 1973 . 
MAGNUS (1970) Inviscid Transonic Flow Over Airfoils; Magnus, R., and Yoshihara, H. AIAA Paper 70-74, 1970.

MAGNUS (1974) Finite Difference Calculations of The NACA 64A410 Airfoil Oscillating Sinusoidally In Pitch At $M=.72$; Magnus, R., Yoshihara, H.; General Dynamics Corp., San Diego, Cal if., August 1974, CASD-NSC-74-004, (N74-34463).

MAGNUS (1975) Unsteady Transonic Flows Over An Airfoil; Magnus, R., Yoshihara, H.; AIAA j Vol. 13 No. 12 Dec. 1975 P. 1622-1628, al so AIAA Paper 75-98, 13th Aerospace Sciences Meeting, January 1975, (A75-13307).

MAGNUS (1976) Calculation Of The Transonic Oscillating Flap With 'Viscous' Displacement Effects; Magnus, R. J., Yoshihara, H.; July 1976, American Institute of Aeronautics and Astronautics, Fluid and Plasma Dynamics Conference, 9th, San Diego, Calif., July 14-16, 1976, AIAA Paper 76-327, (A76-39856).

MAGNUS (1977)a Computational Research On Inviscid, Unsteady, Transonic Flow Over Airfoils; Magnus, R. J.; General Dynamics/Convair San Diego, Calif., CASD/LVP-77-010, (N77-27079).

MAGNUS (1977)b Calculations of Some Steady Unsteady Transonic Flows About The NACA 64A006 And 64A010 Airfoils;

Magnus, R. J.; July 1977, AFFDL-TR-77-46.

MAGNUS (1977)c The Transonic Oscillating Flap; Magnus, R., and Yoshihara, H.; AGARD CP-226, Unsteady Airloads in Transonic and Separated Flow, April 1978.

MALMUTH (1976) A relaxation Solution for Transonic Flow Over Jet Flapped Airfoils; Malmuth, N.D., and Murphy, W. D., J. AIAA Vol. 14, No. 9, pp 1250-1257, September 1976.

MASON (1978) A Numerical Three-Dimensional Viscous Transonic Wing-Body Analys is And Design Tool; Mason, W., Mackenzie, D. A., Stern, M. A., and Johnson, J. K.; AIAA Paper 78-101; 16th Aerospace Sciences Meeting, January 1978.

MCCROSKEY (1977) Some Current Research In Unsteady Fluid Dynamics The 1976 Freeman Scholar Lecture; McCroskey, M. J.; March 1977, ASME, Transactions, Series I - Journal of Fluids Engineering, Vol. 99, March 1977, P. 8-39, (A77-28483). 
MCCROSKEY (1977)a Some Unsteady Separation Problems For Slender Bodies; McCroskey, W. J.; AGARD Lecture Series 94, Three Dimensional and Unsteady Separation at High Reynolds Numbers, February 1978.

MCCROSKEY (1978)b Prediction Of Unsteady Separated Flow On Oscillating Airfoils; McCroskey, W. J.; AGARD Lecture Series 94 Three Dimensional and Unsteady Separation at High Reynolds Numbers, February 1978.

MCDEVITT (1976) Transonic Flow About a Thick Circular-Arc Airfoil, McDevitt, Levy, L. L., and Deiwert, G. S., J. AIAA Vol. 14, No. 5, pp 606-613, May, 1976.

MEIER (1974) Unsteady Behavior of Transonic Flows; Meier, G. E. A.; Max-Planck-Institut Fuer Stroemungforschung, Goettingen (West Germany), in German, MPIS-MITT-59, (N75-23516).

MELNIK (1973) On Viscous And Wind-Tunnel Wall Effects In Transonic Flows Over Airfoils; Melnik, R. E., Ives, D. C.; July 1973, 6th AIAA Fluid and Plasma Dyn. Conf., Palm Springs, Calif., 16-18 July 1973, (N73-30936).

MIRIN (1975) Difference Methods For Transonic Flows About Airfoils; Mirin, A. A., Burstein, S. Z.; Proceedings of the 4 th International Conference on Numerical Methods in Fluid Dynamics, 277-86, 1975, 24-28 June 1974, Boulder, Colo., USA, Springer-Verlag, Berlin, Germany, (A75-80686).

MORCHOISE (1975) Extension Of The Finite Element Method To A Two-Dimensional Transonic Flow; Morchoise, Y.; presented at the 12th Ecole Natl. Super. De Mecan, Et D'Aerotech./Ceat Colloq. D'Aerodyn. Appl., Poitiers, France, 5-7 November 1975, (N77-10476).

MORETTI (1975) Accuracy And Efficiency In The Numerical Analysis of Transonic Flows; Moretti, G.; Symposium Transsonicum II, Geottingen, West Germany, September 8-13, 1975, proceddings, Berlin, Springer-Verlag, 1976, P. 439-448, (A76-27872).

MORETTI (1976) Circumspect Exploration of Multidimensional Imbedded Shocks; Moretti, Gino; AIAA J Vol. 14 No. 7, Jul 1976, P. 894-899.

MURMAN (1971) Calculation Of Plane Steady Transonic Flows; Murman, E. M., and Cole, J. D.; AIAA J. Vol. No. 1, January 1971. 
MURMAN (1972) A Relaxation Method For Calculating Transonic Flows With Detached Bow Shocks; Murman, Earll M.; Proc. of 3rd International Conf. on Numerical Methods in Fluid Mechanics (Springer-Verlag, 1973).

MURMAN (1972)b Computation of Wall Effects in Ventilated Transonic Wind Tunnels; Murman, E. M., AIAA Paper No. 72-1007, September, 1972.

MURMAN (1973) Analysis of Embedded Shock Waves Calculated by Relaxation Methods, Murman, E. M., Proceeding of the AIAA Computational Fluid Dynamics Conference, July, 1973.

MURMAN (1975) TSFOIL - A Computer Code for Two-Dimensional Transonic Calculations Including Wind-Tunnel Wall Effects and Wave Drag Evaluation; Murman, E. M., Bailey, F. R., and Johnson, M. L., National Aeronautics and Space Administration, Ames Research Center, Moffett Field, California, Aerodynamic Analyses Requiring Advanced Computers, SP-347, Part 2, pp 769-788, 1975.

MURMAN (1977) Study of Design And Analysis Methods For Transonic Flow; Murman, E. M.; Flow Research Inc., Kent, Wash., NASA-CR-152041, (N77-30086).

MURPHY (1977) Relaxation Solution For Transonic Flow Over

Three-Dimensional Jet-Flapped Wings; Murphy, W. D., Malmuth, N. D.; AIAA J Vol. 15 No. 1, January 1977, P. 46-53, also AIAA Paper 76-98, 14th Aerospace Sciences Meeting 1976, (A76-18789).

MYKYTOW (1977)a A Brief Overview Of Transonic Flutter Problems; Mykytow, W. J.; AGARD CP-226 Unsteady Airloads in Separated and Transonic Flow, April 1977.

MYKYTOW (1977)b Evaluation Report of The AGARD SMP Specialists Meeting On Transonic Unsteady Aerodynamics For Aeroelastic Phenomena; Mykytow, W. J., and 0lsen, J. J.; AGARD CP-227, Unsteady Aerodynamics, September 1977.

NAVES (1975) Calculations On Airfoil-Fuselage Combinations In Transonic Flow, Problems Resulting From The Application Of The Finite Element Method; Naves, J.; presented at the 12th Ecole Nat1., Super de Mecan, Et d'Aerotech./Ceat Colloq., D'Aerodyn. Appl., Poitiers, France, 5-7 November 1975, in French, (N77-13004). 
NAZARENKO (1972) Pressure Measurement On A Vibrating Aileron In Transonic Flow; Nazarenko, V. V., Nevezhina, T. P.; April 1972, AKademiia Nauk SSSR, Izvestiia, Mekhanika Zhidkosti I Gaza, March-April 1972, P. 179-185, in Russian, (A72-31026).

NEWMAN (1971) An Annotated Bibliography On Transonic Flow Theory, Newman, P. A., and Allison, D. 0., NASA TMX-2363, September 1971 .

NEWMAN (1976) Influence Of Nonconservative Differencing On Transonic Streaml ine Shapes; Newman, P. A., South, J. C,, Jr.; AIAA J (USA) Vol. 14, No. 8, 1148-9, August 1976, (A77-023983).

NICHOLS (1977) Time Integration Of Unsteady Transonic Flow To A Steady State Solution By The Finite Element Method; Nichols, Raymond John Jr., Naval Postgraduate School Monterey Calif., Master's thesis, (N77-30432).

NIEULAND (1967) Transonic Potential Flow Around a Family of Quasi-Elliptical Aerofoil Sections; Nieuwland, G. Y., NLR TR-T-172, 1967.

NIXON (1977) Perturbation Of A Discontinuous Transonic Flow; Nixon, D.; American Institute of Aeronautics and Astronautics, Aerospace Sciences Meeting, 15th, Los Angeles, Calif., January 25-26, 1977, 8 p., AIAA Paper 77-206, (A77-19898).

NIXON (1978) Calculation of Unsteady Transonic FLows Using The Integral Equation Method; Nixon, D.; AIAA Paper 78-13, 16 th Aerospace Sciences Meeting, January 1978.

NIEUWLAND (1973) Transonic Airfoils - Recent Developments In Theory, Experiment, And Design; Nieuwland, G. Y., Spee, B. M., Van Dyke, M. Vincenti, W. G., Wehausen, J. V.; Annual Review of Fluid Mechanics, Vol. 5, 119-50, 1973, (A73-33247).

OEHMEN (1973) A Computational Procedure For Steady And Unsteady Transonic Flows About Slender Profiles; Oehmen, K. H.; Hannover, Technische Universitaet, Fakultaet Fuer Maschinenwesen, Dr. -Ing, dissertation, 1973, in German, (A74-43253).

OEHMEN (1976) Extension of The Parabolic Method For Transonic Flow: Stationary Flow And Extension of The Parabolic Method For Transonic Flows: Unsteady Flow; Oehmen, K. H., Te ipel, I; ; NASA January 1976, NASA-TT-F-16856, (N76-18384). 
OLSEN (1976) Subsonic And Transonic Flow Over Sharp And Round nosed Nonlifting Airfoils; 0lsen, J. J.; Ph.D. thesis, Ohio State University, Columbus, 1976, (N76-30149).

OLSEN (1978) AGARD Standard Configurations for Aeroelastic Applications of Transonic Unsteady Aerodynamics; 01sen, J. J., AFFDL-TM-78-6, October, 1978.

OL'SHANSKII (1976) Of Forces Acting On An Airfoil Vibrating In A Transonic Flow; 0l'Shanskii, V.Yu., Orel, A. A., Fal'Kovich, S. V.; Vestn. Leningr. Univ. Ser. Mat. Mekh. and Astron. (USSR), No. 4 105-14, October 1976, Russian, (A77-039308).

PHARES (1976) Solution To The Eulerian Equations By The Finite Element Method With An Application To Transonic Flow; Final Report, November 1974 - November 1975; Phares, W. J., Kneile, K. R.; ARO, Inc., AEDC-TR-76-86, (N77-17798).

PLATZER (1972) Transonic Aerodynamics Past Progress And Current Status; Platzer, M. F.; Naval Postgraduate School, Monterey, Calif., December 1972, NPS-57PL72120A.

REED (1976) Comparisons of Flight Measurements With Prediction From Aeroelastic Models In The NASA Langley Transonic Dynamics Tunnel; Reed, Wilmer H., III; Conf. Proc. n $187 \mathrm{Apr} 1976$, 46th Meet of Flight Mech Panel;

Flight/Ground Test Facil, June 9-13, 1975.

RIZZETTA (1977)a Transonic Flutter Analysis of A Two-Dimensional Airfoil; Rizzetta, D. P.; AFFDL TM 77-64-FBR, July 1977.

RIZZETTA (1977)b A Comparative Study of Two Computational Methods For Calculating Unsteady Transonic Flows About Oscillating Airfoils; Rizzetta, D. P.; AFFDL TR-77-118, November 1977.

RIZZETTA (1977)c The Aeroelastic Analysis of A Two-Dimensional Airfoil In Transonic Flow; Rizzetta, D. P.; AFFDL-77-126, December 1971.

RIZZI (1975) Transonic Solutions of The Euler Equations By The Finite Volume Method; Rizzi, A.; Symposium Transsonicum II, Goettingen, West Germany, September 8-13, 1975, proceedings, Berlin, Springer-Verlag, 1976, P. 567-574, $($ A76-27883). 
Finite-Volume Solution of The Euler Equations For Steady Three-Dimensional Transonic Flow; Rizzi, A, Bailey, H.; Proceedings of the 5th International Conference on Numerical Methods in Fluid Dynamics, 247-52, 1976, 28 June - 3 July 1976, Enschede, Netherlands, Springer-Everlag, Berlin, Germany, (A77-055028).

RODDEN (1976) State-Of-The-Art In Unsteady Aerodynamics; Rodden, W. P.; Advisory Group for Aerospace Research and Development, Paris (France), November 1976, 43rd Structures and Materials Panel Meeting, Londong, September 1976, AGARD-R-650, (N77-14997).

ROSE (1977) Calculation of Transonic Flow Over Supercritical Airfoil Sections; Rose, W. C., and Seginer, A.; AIAA Paper 77-681, 10th Fluid and Plasmadynamics Conference, June 1977.

RUBESIN (1976) An Experimental And Computational Investigation of The Flow Field About A Transonic Airfoil In Supercritical Flow With Turbulent Boundary-Layer Separation; Rubesin, M. W., Okuno, A. F., Levy, L. L., Jr., McDevitt, J. B., Seemiller, H. L.; National Aeronautics and Space Adrninistration, Ames Research Center, Moffett Field, Calif., July 1976, NASA-TM-X-73157, (N76-28514).

RUO (1974)a Calculation of Unsteady Transonic Aerodynamics For Oscillating Wings With Thickness; Ruo, S. Y., Yates, E., Carson Jr., Theisen, J. G.; J Aircr Vol. 11 No. 10 Oct 1974 P. 60i-608, also AIAA Paper 73-316, Dynamics Specialist Conference, (A73-25547).

RUO (1974)b Calculation of Unsteady Transonic Aerodynamics For Oscillating Wings With Thickness (Computer Program); Marietta, Ruo, S. Y.; Lockheed-Georgia Co., September 1974, NASA-CR-132477, (N74-33427).

RUO (1975) Calculation Of Unsteady Transonic Aerodynamics For Oscillating Wings With Thickness; Ruo, S. Y., Theisen, J. G.; Lockheed-Georgia Co., Marietta, NASA Contract Rep. CR-2259, June 1975, (N75-28030).

SAVKAR (1976) A Note On Transonic Flow Past A Thin Airfoil Oscillating In A Wind Tunnel; Savkar, S. D.; J. Sound and Vib., (GB), Vol. 46, No. 2, 195-2097, 22 May 1976, (A76-64812). 
SCHMIDT (1975) Some Results Using Relaxation Methods For Two- And Three-Dimensional Transonic Flows; Schmidt, W., Rohlfs, S., Vaning, R.; proceedings of the 4 th International Conference on Numerical Methods in Fluid Dynamics, 364-72, 1975, 24-28 June 1974, Boulder, Colo., USA, Springer-Verlag, Berlin, Germany, (A75-42225).

SCHMIDT (1976) Numerical Simulation Of Three-Dimensional Transonic Flow Including Wind Tunnel Wall Effects; Schmidt, W., Stock, H. -W, Fritz, W.: AGARD Conference Proceedings No. 210 on Numerical Methods and Wind Tunnel Testing, 16/1-8, 1976, 23-24 June 1976, Rhode-St-Genese, Belgium, (N77-11984).

SEEBASS (1974) Improvements To Embedded Shock Wave Calculations For Transonic Fluw Applications To Wave Drag And Pressure Rise Predictions; Seebass, A. R.; Cornell Univ., Ithaca, N.Y., USA, NASA-CR-139487, (N74-30629).

SEEBASS (1976) Numerical Simulation Of Small Perturbation Transonic Flows; Final Report, 1 September 1973 - 31 December 1975; Seebass, A. R., Yu, N. J.; Cornell Univ., Ithaca, N.Y., April 1976, NASA-CR-147155, (N76-22490).

SEEBASS (1977) Unsteady Transonic Flow Computations; Seebass, A. R., Yu, N. J. Fung, K. Y.; AGARD Cp-227, Unsteady Aerodynamics, September 1977.

SEEGMILLER (1978) Steady And Unsteady Transonic Flow; Seegmiller, H. L., Marvin, J. G., and Levy, L. L.; AIAA Paper 78-160, 16th Aerospace Sciences Meeting, January 1978.

SEGINER (1977) An Approximate Calculation of The Strong Interaction On A Transonic Airfoil; Seginer, A., Rose, W. C.; January 1977. American Institute of Aeronautics and Astronautics, Aerospace Sciences Meeting, 15th, Los Angeles, Calif., January 24-26, 1977, AIAA Paper 77-210, (A77-19900).

SHANKAR (1978) Computational Transonic Airfoil Design in Free Air and a Wind Tunnel; Shanker, V., Malmuth, N. D., and Cole, J. D., AIAA Paper 78-103, January, 1978.

SILLS (1968) Computation of Inviscid, Two-Dimensional, Transonic Flows Using A Time-Dependent Finite-Difference Method; Sills, J. A.; General Dynamics/Fort Worth, Tex., December 1968, GDC-ERR-FW-806, (N72-70834).

SILLS (1972) Relaxation Solutions For Inviscid Transonic Airfoil Flow Fields; Sills, J. A.; Generai Dynamics, Fort Worth Div., December 1972, ERR-RW-1359. 
SOUTH (1976)a Comments On Difference Schemes For The

Three-Dimensional Transonic Small-Disturbance Equation For Swept Wings; South, J. C., Jr.; National

Aeronautics and Space Administration, Langley Research Center, Langley Station, Va., July 1976, NASA-TM-X-71980, (N76-28189).

SOUTH (1976)b The Multigrid Method: Fast Relaxation; South, J. C., Jr.; Brandt, A., Advan. in Eng. Sci., Vol. 4, P. 1359-1369, (N77-10352).

SPREITER (1971) Calculative Techniques For Transonic Flows About Certain Classes of Airfoils And Slender Bodies; Spreiter, J. R., Stahara, S. S.; Nielsen Engineering and Research, Inc., Mountain View, Calif., April 1974, NASA-CR-1722, (N71-23133).

SPREITER (1975) Unsteady Transonic Aerodynamics, An Aeronautics Challenge; Spreiter, John R., Stahara, Stephen S.; Unsteady Aerodyn. Symp. Proc. Univ of Ariz., Tucson, March 18-20, 1975 Vol. 2 P. 553-581, Publ by Ariz Board of Regents, Tucson, 1975.

SPREITER (1976) Developments In Transonic Steady And Unsteady Flow Theory; Spreiter, J. R., Siahara, S. S.; International Council of the Aeronautical Sciences, Congress, 10th, Ottawa, Canada, October 3-8, 1976, ICAS Paper 76-06.

STAHARA (1973) Development of A Nonlinear Unsteady Transonic Flow Theory; Stahara, S. S., Spreiter, J. R., Nielsen Enginering and Research, Inc., Mountain View, Calif., June 1973 , NASA-CR-2258.

STAHARA (1976)a Research On Unsteady Transonic Flow Theory; Stahara, Stephen S., Spreiter, John R.; Nielsen Engineering and Research Inc Mountain View Calif., NEAR-TR-132.

STEGER (1973)a Shock Waves And Drag In The Numerical Calculation Of Compressible; Irrotational Transonic Flow; Steger, J. L., Baldwin, B. S.; AIAA J (USA), Vol. 11, No. 7, 903-4, July 1973, (A73-78984).

STEGER (1973)b A Finite Difference Method for Transonic Airfoil Design; Stegar, J. L., and Klineberg, J. M., J. AIAA, Vol. 11, p 628, 1973.

STEGER (1977) Implicit Finite Difference Simulation of Flow About Oscillating Geometrics With Application To Airfoils; Steger, J. L.; AIAA Paper 77-665, 10th Fluid and Plasmadynamics Conference, June 1977. 
THIEDE (1976) Prediction Method For Steady Aerodynamic Loading On Airfoils With Separated Transonic Flow; Thiede, P. G.; AGARD Conf. Proc. $n$ 204, Predict of Aerodyn Loading, from the Fluid Dyn Panel Symp., February 1977, 1-16. 12, (N77-20005).

TIJDEMAN (1973)a On The Prediction Of Aerodynamic Loads On Oscillating Wings In Transonic Flow; Tijdeman, H., Zwaan, R. J.; National Aerospace Lab., Amsterdam (Netherlands), June 1973 , presented at the 36 th meeting of the Struct. and Mater. Panel, Milan, 4 April 1973, NLR-MP-73026-U, (N74-33442).

TIJDEMAN (1973)b Results of Pressure Measurements On An Airfoil with Oscillating Flap In Two-Dimensional High Subsonic And Transonic Flow (Zero Incidence And Zero Mean Flap Position); Tijdeman, H., Schippers, P.; National Aerospace Lab., Amsterdam (Netherlands), Flight Dynamics Div., July 1973, NLR-TR-73078-U, (N74-33443).

TIJDEMAN (1975)a On The Motion of Shock Waves On An Airfoil with Oscillating Flap In Two-Dimensional Transonic Flow; Tijdeman, H.; National Aerospace Lab., Amsterdam (Netherlands), Fluid Dynamics Div., 14 March 1973, NLR-TR-75038-U, (N77-15994).

TIJDEMAN (1975)b High Subsonic And Transonic Effects In Unsteady Aerodynamics; Tijdeman, H.; National Aerospace Lab., Amsterdam (Netherlands), NLR-TR-75079-U, (N77-13014).

TIJDEMAN (1976)a Some Remarks On Unsteady Transonic Flow; Tijdeman, H.; Nat 1 Aerosp Lab NLR, Amsterdam, Neth, AGARD Rep n 645 March 1976, Unsteady Aerodyn, for Meet., Goettingen, Ger., May 1975, (N76-24248).

TIJDEMAN (1976)b On The Unsteady Aerodynamic Characteristics of Oscillating Airfoils In Two-Dimensional Transonic Flow; Tijdeman, H.; National Aerospace Lab., Amsterdam (Netherlands, NLR-MP-76003-U, presented at the ONR Transonic Flow Conf., Los Angeles, 30-31 March 1976, (N77-18060).

TIJDEMAN (1976)c High Subsonic And Transonic Effects In Unsteady Aerodynamics; Tijdeman, H.; AGARD Rep $n 636$ January 1976 P. 1-29.

TIJDEMAN (1977)a Unsteady Airloads On An Oscillating Supercritical Airfoil; Tijdeman, H., Schippers, P., Pearson, A. J.; National Aerospace Lab., Amsterdam, (Netherlands), in AGARD Unsteady Airloads in Separated and Transonic Flow, Apri1 1977, (N77-31085). 
TIJDEMAN (1977)b Remarks On The Transonic Flow Around Oscillating Airfoils; Tijdeman, H.; AGARD CP-227, Unsteady Aerodynamics, September 1977.

TIJDEMAN (1977)c Investigations of The Transonic Flow Around Oscillating Airfoils; Tijdeman, H.; NLR TR 77090-U, 1977.

TRACI (1974) Small Disturbance Transonic Flows About Oscillating Airfoils; Technical Report, July 1973 - January 1974, Wright-Patterson AFB, Ohio, June 1974, AFFDL-TR-74-37, (x75-73639).

TRACI (1975) Small Disturbance Transonic Flows About Oscillating Airfoils And Planar Wings; Traci, R. M., Albano, E. D., Farr, J. L., Jr.; Final Report, June 1974 - June 1975,, August 1975, AFFDL-TR-75-100, (X76-77844).

TRACI (1976) Perturbation Method For Transonic Flows About Oscillating Airfoils; Traci, R. M., Albano, E. D., Farr, J. L., Jr.; AIAA J Vol. 14 No. 9, September 1976, P. $1258-1265$, also AIAA Paper $75-877$, 8th Fluid and Plasmadynamic Conference, June 1975.

TRIEBSTEIN (1969) Unsteady Pressure Distribution Measurements On A Harmonically Oscillating Wing In The Subsonic And Transonic Speed Range; Triebstein, H.; July 1969, the DGLR Ann. Meeting, Bremen, 22-24, September 1969, AVA-FB-6913, (N70-23832 \& A70-15184).

TURBATU (1975) Perturbation Potential For Aperiodic Unsteady Transonic Flows; Turbatu, S.; Symposium Transsonicum II, Goettingen, West Germany, September 8-13, 1975, proceedings, Berlin, Springer-Verlag, 1976, P. 33-40, in German, (A76-27828).

TURBATU (1976) Perturbation Potential For A Thin Wing Of Small Span; Turbatu, S; Bulletin Mathematique, Vol. 18, (1974), No. $3-4,1976$, P. 415-423, in German.

VANDERVOOREN (1975) Remarks On The Suitability Of Various Transonic Small Perturbation Equations To Describe Three-Dimensional Transonic Flow - Examples of Computations Using A Fully-Conservative Rotated Difference Scheme; Van Der Vooren, J., Sloof, J. W., Huizing, G. H., Van Essen, A.; Symposium Transsonicum II, Goettingen, West Germany, Septebmer 8-13, 1975, proceedings, Berlin, Springer-Verlag, 1976, P. 557-566, (N77-15992). 

Transsonicon II, Goettingen, West Germany, September $8-13,1975$, proceedings, Berlin, Springer-Verlag, 1976, P. 281-288, (A76-27856).

WARMING (1975) Upwind Second-Order Difference Schemes And Applications In Unsteady Aerodynamic Flows; Warming, R. F., Beam, R. M.; Computational Fluid Dynamics Conference, 2nd, Hartford, Conn., June 19, 20,1975, proceedings, American Institute of Aeronautics and Astronautics, Inc., 1975, P. 17-28, (A75-34181).

WARMING (1976) Upwind Second-Order Difference Schemes And Applications In Aerodynamic Flows; Warming, R. F., Beam, Richard M.; AIAA J Vol. 14 No. 9 September 1976 P. 1241-1249.

WEATHERILL (1975) Computation of The Transonic Perturbation Flow Fields Around Two- And Three-Dimensional Oscillating Wings; Final Report; Weatherill, W. H., Ehlers, F. E., Sebastian, J. D., Boeing Commercial Airplane Co., Seattle, Wash., NASA-CR-2599, (N76-17072).

WEATHERILL (1976) Computation of The Transonic Perturbation Flow Field Around Two- And Three-Dimensional Oscillating Wings; Weatherill, W. H., Ehlers, F. E., Sebastian, J. D.; American Institute of Aeronautics and Astronautics, Aerospace Sciences Meeting, 14th, Washington, D.C., January 26-28, 1976, AIAA Paper 76-99, (A76-18790)'

WEATHERILL (1977) Application of A Finite Difference Method To The Analys is of Transonic Flow Over Oscillating Airfoils And Wings; Weatherill, W. H., Sebastian, J. D., Ehlers, F. E.; Boeing Commercial Airplane Co., Seattle, Wash., in AGARD CP-226 Unsteady Airloads in Separated and Transonic Flow, 13 p., April 1977, (N77-31090).

WU (1974)

Approximate Solution of Unsteady Transonic Flow Problems; Technical Report; Tullahoma, Wu, J. M., Kimble, K. R.; June 1974, June 1973 - January 1974, Tennesse Univ., Space Inst., AFFDL-TR-74-32, (N74-34718).

WU (1976) A Survey Of Transonic Aerodynamics; Wu, J. M., Moulden, T. H.; July 1976, 294 Rees, American Institute of Aeronautics And Astronautics, Fluid and Plasma Dynamics Conference, 9th, San Diego, Calif., July 14-16, 1976, AIAA Paper 76-326, (A76-39855). 
YOSHIHARA (1973) A Survey Of Computational Methods For 20 and 30 Transonic Flows With Shocks; Yoshihara, H.; AGARD Lecture Series No. 64, Advances in Numerical Fluid Dynamics, 1973, 5-9 March 1973, Brussels, Belguim, (N74-22920, A75-17959).

YU (1975) Inviscid Transonic Flow Computations With Shock Fitting; Yu, N. J., Seebass, A. R., Arizona Univ., Tuc son, NASA-CR-145430.

YU (1977) An Implicit Shock-Fitting Scheme For Unsteady Transonic Flow Computations; Tucson, Yu, N. J., Seebass, A. R., Ballhaus, W. F.; Arizona Univ., Computational Fluid Dynamics Conference, 3rd, Albuquerque, N. Mex., June 17, 28, 1977, Technical Paper, American Institute of Aeronautics and Astronautics, AIAA 77-633. 